\title{
Knock-In Mouse Models to Investigate the Functions of Opioid Receptors in vivo
}

\author{
Jade Degrandmaison ${ }^{1,2,3}$, Samuel Rochon-Haché1,2,3, Jean-Luc Parent ${ }^{1 \star}$ and \\ Louis Gendron $2,4 *$ \\ ${ }^{1}$ Centre de Recherche du Centre Hospitalier Universitaire de Sherbrooke, Département de Médecine, Institut \\ de Pharmacologie de Sherbrooke, Faculté de Médecine et des Sciences de la Santé, Université de Sherbrooke, Sherbrooke, \\ QC, Canada, ${ }^{2}$ Centre de Recherche du Centre Hospitalier Universitaire de Sherbrooke, Département \\ de Pharmacologie-Physiologie, Institut de Pharmacologie de Sherbrooke, Faculté de Médecine et des Sciences de la Santé, \\ Université de Sherbrooke, Sherbrooke, QC, Canada, ${ }^{3}$ Quebec Network of Junior Pain Investigators, Sherbrooke, QC, \\ Canada, ${ }^{4}$ Quebec Pain Research Network, Sherbrooke, QC, Canada
}

\section{OPEN ACCESS}

Edited by:

Yamina Berchiche,

Dr. GPCR, United States

Reviewed by:

Tuan Trang,

University of Calgary, Canada

Rita Bardoni,

University of Modena and Reggio

Emilia, Italy

*Correspondence:

Louis Gendron

Louis.Gendron@USherbrooke.ca

Jean-Luc Parent

Jean-Luc.Parent@USherbrooke.ca

Specialty section:

This article was submitted to

Cellular Neurophysiology,

a section of the journal

Frontiers in Cellular Neuroscience

Received: 02 November 2021

Accepted: 04 January 2022

Published: 31 January 2022

Citation:

Degrandmaison J,

Rochon-Haché S, Parent J-L and Gendron L (2022) Knock-In Mouse Models to Investigate the Functions

of Opioid Receptors in vivo.

Front. Cell. Neurosci. 16:807549.

doi: 10.3389/fncel.2022.807549
Due to their low expression levels, complex multi-pass transmembrane structure, and the current lack of highly specific antibodies, the assessment of endogenous G proteincoupled receptors (GPCRs) remains challenging. While most of the research regarding their functions was performed in heterologous systems overexpressing the receptor, recent advances in genetic engineering methods have allowed the generation of several unique mouse models. These animals proved to be useful to investigate numerous aspects underlying the physiological functions of GPCRs, including their endogenous expression, distribution, interactome, and trafficking processes. Given their significant pharmacological importance and central roles in the nervous system, opioid peptide receptors $(\mathrm{OPr})$ are often referred to as prototypical receptors for the study of GPCR regulatory mechanisms. Although only a few GPCR knock-in mouse lines have thus far been generated, OPr are strikingly well represented with over 20 different knock-in models, more than half of which were developed within the last 5 years. In this review, we describe the arsenal of $\operatorname{Pr}$ (mu-, delta-, and kappa- opioid), as well as the opioidrelated nociceptin/orphanin FQ (NOP) receptor knock-in mouse models that have been generated over the past years. We further highlight the invaluable contribution of such models to our understanding of the in vivo mechanisms underlying the regulation of $\mathrm{OPr}$, which could be conceivably transposed to any other GPCR, as well as the limitations, future perspectives, and possibilities enabled by such tools.

Keywords: G protein-coupled receptor (GPCR), opioid receptor, knock-in (KI) mice, nociceptin receptor, mouse model, fluorescent protein, in vivo

\section{INTRODUCTION}

Characterized by a common topology exhibiting an extracellular $N$-terminal domain, seven hydrophobic membrane $\alpha$-helices and a cytosolic $C$-terminus, G protein-coupled receptors (GPCR) form the largest family of transmembrane proteins (Hauser et al., 2017). With over 800 different members, these receptors can respond to a vast array of ligands, including peptides, lipids, photons, neurotransmitters, and hormones to fine-tune virtually every physiological system (Hauser et al., 2017). Most importantly, GPCRs represent long-standing powerful therapeutic targets with approximately $34 \%$ of the currently marketed drugs targeting these transmembrane 
proteins (Hauser et al., 2017; Kooistra et al., 2021). Despite their significant clinical importance, the study of GPCRs in vivo has been impaired by several challenges. Indeed, their relatively low endogenous expression levels, their complex transmembrane structure and the lack of specific and potent antibodies have contributed to limit our understanding of their functions in physiologically relevant conditions (Michel et al., 2009; Jo and Jung, 2016). The design of new tools and approaches to study GPCRs in vivo is therefore crucial to develop and improve current therapeutics.

Belonging to the class-A GPCRs, the opioid receptors family (OPr), which comprises the mu ( $\mu$, MOPr), delta $(\delta$, DOPr) and kappa $(\kappa$, KOPr) subtypes, share between 59 and $63 \%$ of amino acids sequence identity (Pathan and Williams, 2012; Degrandmaison et al., 2021). Moreover, the nociceptin receptor (NOPr), originally referred to as the $\kappa$ type 3 opioid receptor, is also closely related to the OPr family (Borsodi et al., 2019). Opioid and nociceptin receptors are predominantly expressed throughout the nervous system where they specifically regulate a wide range of physiological effects, mostly associated with the nociception-, stress-, mood-, and reward-related pathways. Although the pharmacological profile exhibited by the NOPr is relatively more complex (discussed in section "Nociceptin/orphanin FQ receptor knock-in mouse lines"), ligand-mediated activation of these receptors results in analgesia, as well as other distinct physiological responses (Reinscheid et al., 1995; Grisel et al., 1996; Mogil et al., 1996a,b; Xu et al., 1996; Yamamoto et al., 1997; Mogil and Pasternak, 2001; Borsodi et al., 2019). Given their significant clinical relevance, $\mathrm{OPr}$ are established as a model family of receptors to investigate the cellular mechanisms underlying the regulation of GPCRs, therefore placing them at the forefront of recent advances in the GPCR field.

As for other members of the GPCR family, initial research conducted on $\mathrm{OPr}$ and their endogenous ligands relied on

Abbreviations: $\alpha_{2 a}$-AR, alpha-2a adrenergic receptor; AAV, adeno-associated virus; $\beta$ arr2, beta-arrestin 2; BRET, bioluminescence resonance energy transfer; CFA, complete Freund's adjuvant; $\mathrm{CPu}$, caudate putamen; CreER, Cre recombinase-modified estrogen receptor; CRISPR/Cas9, clustered regulatory interspaced short palindromic repeats and its associated protein-9; DAMGO, [D-Ala ${ }^{2}, \mathrm{~N}-\mathrm{Me}-\mathrm{Phe}^{4}, \mathrm{Gly}^{5}$-ol]-enkephalin; DH, dorsal hippocampus; dKI, double knock-in; DOPr, delta-opioid receptor; DREADD, designer receptor exclusively activated by designer drug; DRG, dorsal root ganglion; eGFP, enhanced green fluorescent protein; (ETC)-CLARITY, electrophoretic tissue clearingCLARITY; FP, fluorescent protein; FRT, flippase recognition target; GPCR, G protein-coupled receptor; GRK2, G protein-coupled receptor kinase 2; HA, hemagglutinin; HEK293, human embryonic kidney cells; IPN, interpeduncular nucleus; KI, knock-in; KO, knock-out; KOPr, kappa-opioid receptor; LAMP-I, lysosome-associated membrane glycoprotein-1; MHb, medial habenula; MOPr, mu-opioid receptor; MS, mass spectrometry; mTagBFP2, monomeric Tag blue fluorescent protein; mTFP1, monomeric teal fluorescent protein 1; N/OFQ, nociceptin/orphanin FQ; NAc, nucleus accumbens; NMDA, N-Methyl-Daspartate receptor; NOPr, nociceptin/orphanin FQ receptor; OPr, opioid peptide receptors; PAG, periaqueductal gray; PTM, post-translational modification; RET, resonance energy transfer; S1P1, sphingosine-1-phosphate receptor 1; SNC80, 4-[(R)-[(2S,5R)-4-allyl-2,5-dimethylpiperazin-1-yl](3-methoxypheny) methyl]$N, N$-diethylbenzamide; SNI, spared nerve injury; SNL, spinal nerve ligation; SNP, single nucleotide polymorphism; snRNAseq, single-nucleus RNA sequencing; T2A, Thosea asigna virus 2A-like peptide; TALEN, transcription activator-like effector nuclease; tdTomato, tandem dimer Tomato; Tm, tamoxifen; VH, ventral hippocampus; VTA, ventral tegmental area; WT, wildtype; YFP, yellow fluorescent protein. the partial deletion of targeted genes within an animal, generating knock-out (KO) models. This "loss-of-function" genetic approach has revolutionized the study of opioid functions in vivo by offering a complementary approach to classical pharmacology. The precise deletion of each component of the endogenous opioid and NOPr-N/OFQ systems has indeed shed new light on their specific individual contribution to pathophysiological states such as acute and chronic pain (see Kieffer, 1999; Dierich and Kieffer, 2004; Nadal et al., 2013; Maldonado et al., 2018 for reviews). Further progress regarding genetically engineered mice has then led to the development of transgenic mouse lines, which are characterized by the rather random integration of an exogenous gene sequence (Doyle et al., 2012; Ceredig and Massotte, 2015). Although these models proved themselves useful for specific applications, several drawbacks have been associated to such an approach, including the overexpression of the encoded protein as compared to wildtype (WT) animals and the improper insertion of the transgene (e.g., within undesired tissue or genomic regions) (Doyle et al., 2012; Ceredig and Massotte, 2015). In recent years, an alternative strategy aiming to specifically introduce or modify a gene of interest has emerged. The generation of knock-in (KI) mouse strains consists of specifically inserting or modifying a gene at the locus of interest, thus overcoming most of the disadvantages associated with transgenic models. Such a strategy further opened the path to a myriad of possibilities regarding the creation of unique mouse lines. The study of OPr has also significantly benefited from this expanding technology as demonstrated by the 13 novel OPr KI mouse strains that have been generated between 2019-2021, for a total of more than 20 different OPr and NOPr KI mice published as of to date. These OPr-based KI mice have been designed according to diverse strategies including the fusion of the selected receptor with a fluorescent protein (FP) or a small epitope-tag [e.g., hemagglutinin (HA) or FLAG], as well as the insertion of a Cre recombinase. In the following sections, we describe the currently available KI mouse lines that have been created for NOPr and each OPr subtypes, as well as their contribution to our understanding of the endogenous molecular and physiological roles of the opioid and nociceptin systems. We also further discuss the limitations and the perspectives related to the study of OPr in animal models.

\section{$\mu$-Opioid Receptor Knock-In Mouse Lines}

Undoubtedly the most extensively studied opioid receptor, the MOPr is the primary target of most clinical opioid therapeutics, including morphine, fentanyl and codeine (Pathan and Williams, 2012). Although activation of the MOPr has been associated with yet unmatched analgesic properties, its agonists are also accountable for a broad range of serious adverse effects such as tolerance, dependence, addiction, constipation, and respiratory depression when used over an extended period of time (Morgan and Christie, 2011). Current research efforts on the MOPr therefore mainly focus on deciphering the cellular and physiological mechanisms involved in the development of such adverse effects, as well as the design of better-tolerated 


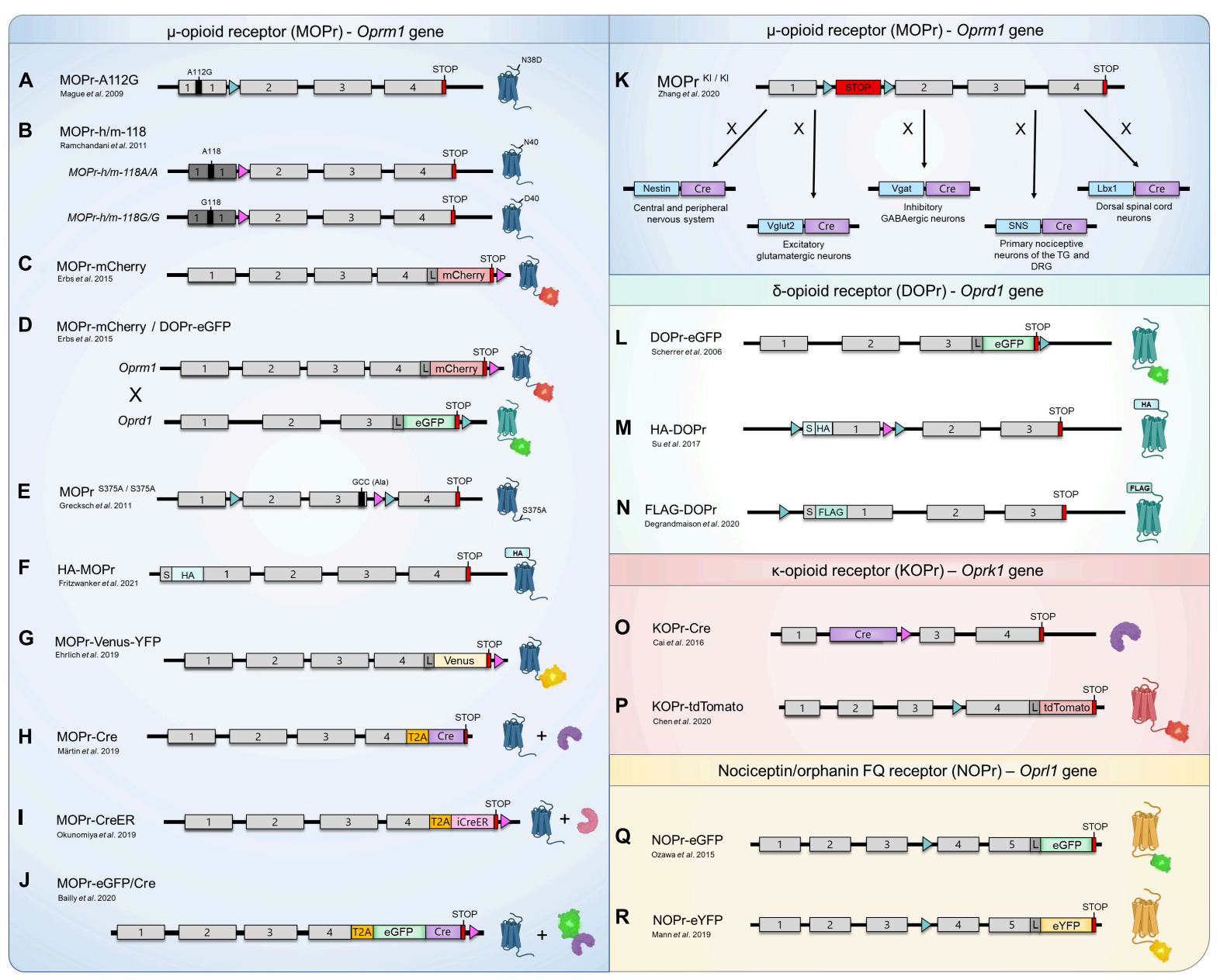

FIGURE 1 | Opioid and nociceptin receptors knock-in mouse lines. (A-R) Schematic representation of the design of currently available KI mouse models for the study of MOPr (blue), DOPr (green), KOPr (red), and NOPr (yellow). The sequence corresponding to the mouse (gray boxes) or human (dark gray boxes) exons, START codons (S), STOP codons (red boxes), loxP sites (aqua triangles), FRT sites (pink triangles), as well as the sequences encoding for various fluorescent proteins (eGFP, mCherry, tdTomato, Venus and eYFP), epitope-tag sequences (FLAG, HA), linker (L; encoding for Gly-Ser-lle-Ala-Thr), Cre recombinase (Cre, purple boxes), inducible Cre recombinase-modified estrogen receptor (iCreeER, pink box) and the Thosea asigna virus 2A-like peptide (T2A, orange boxes) are identified. The schematic representation is not to scale. (K) Cre-mediated recombination can also occur in other regions than those indicated.

analgesics for chronic pain management. In recent years, a wide variety of MOPr KI mouse lines has been generated. As described below, these animals have provided useful insights regarding the regulation and the functions of this major pharmacological target.

More than a decade ago, Mague et al. (2009) have designed a MOPr KI mouse line harboring the A112G mutation, corresponding to a single nucleotide polymorphism (SNP) previously identified in the human MOPr (A118G in humans). With a prevalence of $15-30 \%$ in Europeans and $49-60 \%$ in individuals of Asian ancestry, this common point mutation resulting in the replacement of an asparagine for an aspartic acid (N40D in humans) at a potential $N$-glycosylation site has been associated with altered responses to opioid-induced analgesia, as well as to distinct phenotypes related to opioid, alcohol and nicotine addictions (Lerman et al., 2004; Ray and Hutchison, 2004, 2007; Kreek et al., 2005; Chou et al., 2006; Drakenberg et al., 2006; van den Wildenberg et al., 2007;
Anton et al., 2008; Sia et al., 2008; Mague et al., 2009; Huang et al., 2012). Since several discrepancies had been observed while investigating the MOPr-N40D variant in heterologous expression systems, the authors used homologous recombination to specifically introduce the A112G mutation within the exon 1 of the Oprm1 gene, resulting in a mouse line expressing the corresponding mutated MOPr-N38D (Figure 1A; Mague et al., 2009). Behavioral assays performed indicated that animals presenting the G112 allele exhibited reduced MOPr expression (mRNA and protein levels), as well as a reduction of the antinociceptive and hyperlocomotor effects induced by acute morphine treatment (Table 1; Mague et al., 2009). Interestingly, the authors also reported sex-specific reductions of the morphine-mediated rewarding properties and the aversive components of naloxone-precipitated morphine withdrawal with only females demonstrating an altered behavioral response (Mague et al., 2009). In a following study carried out by 
TABLE 1 | Characterization of the expression levels, subcellular localization and internalization of genetically modified opioid receptors from KI mice.

\begin{tabular}{|c|c|c|c|}
\hline KI mouse line & Receptor density (vs. WT or CTRL mice) & Subcellular localization and internalization & References \\
\hline MOPr-A112G & Lower in $\mathrm{G} / \mathrm{G}$ animals & $\mathrm{N} / \mathrm{A}$ & $\begin{array}{l}\text { Mague et al., 2009; Wang } \\
\text { et al., } 2012\end{array}$ \\
\hline MOPr-h/m-118G/G, MOPr-h/m-118A/A & Similar between $G / G$ and $A / A$ mice & $\mathrm{N} / \mathrm{A}$ & Ramchandani et al., 2011 \\
\hline MOPr-mCherry & Slightly higher & $\begin{array}{l}\text { Predominant intracellular localization. } \\
\text { DAMGO-mediated internalization is detectable } \\
\text { in primary hippocampal neurons }\end{array}$ & Erbs et al., 2015 \\
\hline MOPr-Cre and MOPr-CreER & $\mathrm{N} / \mathrm{A}$ & $\mathrm{N} / \mathrm{A}$ & $\begin{array}{l}\text { Märtin et al., 2019; } \\
\text { Okunomiya et al., } 2020\end{array}$ \\
\hline MOPr-eGFP-Cre & N/A, but similar mRNA levels & $\mathrm{N} / \mathrm{A}$ & Bailly et al., 2020 \\
\hline HA-MOPr & Lower ( 50\% less in KI mice) & $N / A$ & Fritzwanker et al., 2021 \\
\hline MOPr-Venus-YFP & N/A, but similar mRNA levels & $\begin{array}{l}\text { Internalization of MOPr-Venus-YFP in DRG } \\
\text { neurons following treatments with DAMGO and } \\
\text { Met-Enk, but not buprenorphine nor PZM21. }\end{array}$ & Ehrlich et al., 2019 \\
\hline MOPr S375A/S375A & Similar & Similar subcellular distribution as WT. & Grecksch et al., 2011 \\
\hline DOPr- eGFP & Higher ( 1.5-fold) & $\begin{array}{l}\text { Predominant PM localization. SNC80 induces } \\
\text { endocytosis in vivo and in primary CPu } \\
\text { neurons. }\end{array}$ & Scherrer et al., 2006 \\
\hline HA-DOPr & $\mathrm{N} / \mathrm{A}$ & $\mathrm{N} / \mathrm{A}$ & Su et al., 2017 \\
\hline FLAG-DOPr & Similar & $\mathrm{N} / \mathrm{A}$ & $\begin{array}{l}\text { Degrandmaison et al., } \\
2020\end{array}$ \\
\hline KOPr-Cre & $N / A$ & $\mathrm{~N} / \mathrm{A}$ & Cai et al., 2016 \\
\hline KOPr-tdTomato & Higher ( 12-fold) & $\begin{array}{l}\text { U50,488 treatment induces translocation of } \\
\text { KOPr-tdT from PM to intracellular space. }\end{array}$ & Chen et al., 2020 \\
\hline NOPr- eGFP & Higher ( 2.8-fold) & $\begin{array}{l}\text { Predominant intracellular localization, but some } \\
\text { PM-localized NOPr are also observed. N/OFQ } \\
\text { mediates internalization in primary neurons*. }\end{array}$ & Ozawa et al., 2018 \\
\hline NOPr- eYFP & $\mathrm{N} / \mathrm{A}$ & $\begin{array}{l}\text { In vivo internalization in primary neurons with } \\
\text { various compounds including agonist N/OFQ. }\end{array}$ & Mann et al., 2019 \\
\hline
\end{tabular}

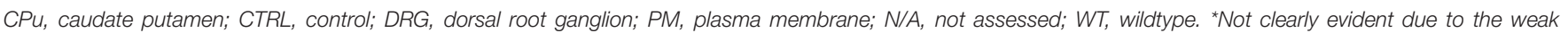
expression of NOPr-eGFP at the neuronal PM.

Wang et al. (2012), binding of the radiolabeled MOPr agonist $\left[{ }^{3} \mathrm{H}\right]$-DAMGO revealed that MOPr-A112G expression is reduced in several, but not all, brain regions as compared to WT MOPr (Table 1). Whereas no significant difference in the binding of $\left[{ }^{3} \mathrm{H}\right]$-DAMGO in the caudate putamen $(\mathrm{CPu})$ and hippocampus was measured, higher expression of MOPr was observed in the cingulate, motor and insular cortices, amygdala, nucleus accumbens (NAc) core and shell, periaqueductal gray (PAG), ventral tegmental area (VTA) and thalamus of A/A mice (Wang et al., 2012). While most of these regions (i.e., amygdala, anterior cingulate, and insular cortices) are often referred to as the "pain matrix" of the brain, others, including the PAG, are known to be directly involved in opioid antinociception (Yaksh and Rudy, 1976; Ingvar, 1999; Brooks and Tracey, 2005). These results are therefore consistent with behavioral experiments indicating that mice carrying the G112 allele present a reduced antinociceptive response to morphine when compared to A/A mice (Mague et al., 2009; Wang et al., 2012).

It has been suggested that the region-specific differences in MOPr expression levels between A112 and G112 mice might be attributed to the variation in $N$-glycan types and contents across the brain (Wang et al., 2012). As reported previously, $N$-glycosylation of GPCRs, a post-translational modification (PTM) mainly occurring in the ER and Golgi apparatus, plays an important role in the correct folding and maturation of the receptors, thereby impacting their neuronal membrane density (Goth et al., 2020; Lemos Duarte and Devi, 2020; Degrandmaison et al., 2021). Presumably due to the differential expression of glycosyltransferases throughout the mouse brain, $N$-glycosylation of MOPr has been shown to be variable depending on the brain areas (Matsuhashi et al., 2003; Huang et al., 2008, 2012). For example, Huang et al. (2008) have observed that the WT MOPr is differentially modified by $N$-glycans in the $\mathrm{CPu}$ vs. the thalamus of the rat and mouse, thus suggesting brain region-specific $N$-glycosylation patterns. In a follow-up study, the authors indicated that the A112G mutation (or A118G in human MOPr) led to a reduction in MOPr $N$-glycosylation in the mouse brain and in cultured cells, which highlights the asparagine 38 (or 40) of the MOPr as a probable glycosylation site (Huang et al., 2012). Since sex-differences in $N$-linked glycosylation have been already reported, with males having higher levels of several types of $\mathrm{N}$-glycans, this hypothesis could also possibly explain the results obtained for the variations in MOPr expression levels (Knežević et al., 2009; Stanta et al., 2010). Other factors, such as epigenetic regulation and the hormonal state of the females, might represent possible causes for this sexual dimorphism (Wang et al., 2012).

A few years later, the involvement of this specific polymorphism in alcohol use disorders was further investigated by Ramchandani et al. (2011). In this study, the authors created two humanized KI mice in which the Oprm1 first exon was replaced by the corresponding human sequence in 
order to generate homozygous mouse lines carrying each one of the variants (i.e., G/G or A/A) (Ramchandani et al., 2011; Figure 1B). These mice, henceforth referred to as MOPr-h/m118A/A (major 118A allele) and MOPr-h/m-118G/G (minor $118 \mathrm{G}$ allele, corresponding to the SNP) showed distinctive striatal dopamine responses when submitted to an alcohol challenge, thus suggesting that the A118G variation represents a genetic determinant possibly modulating alcohol reward (Ramchandani et al., 2011). Interestingly, as opposed to the results reported by Wang et al. (2012) no differences in $\left[{ }^{3} \mathrm{H}\right]$-DAMGO binding have been observed in the dorsal striatum, NAc and VTA of G118 and A118 mice (Ramchandani et al., 2011; Table 1). Although it was suggested that the replacement of the Oprm 1 exon 1 with the homologous human sequence could affect MOPr protein expression and/or maturation, the exact cause explaining this discrepancy remains to be investigated.

Erbs et al. (2015) have generated a MOPr-mCherry KI mouse line. The design used homologous recombination to replace the Oprm1 STOP codon with the sequence encoding the gene for the red protein mCherry followed by an FRT flanked neomycin resistance gene. The resistance gene was then excised by a FLP recombinase targeting the FRT sites, resulting in a mouse expressing MOPr fused with mCherry at its $C$-terminus (Figure 1C). In a concomitant study, the same group mapped the expression of MOPr-mCherry within the habenular complex, revealing a strong expression of the receptor in the medial habenula (MHb), fasciculus retroflexus and interpeduncular nucleus (IPN) (Gardon et al., 2014). Since the $\mathrm{MHb}$ has been previously recognized as a region mediating the analgesic and rewarding properties of opioids, the expression of MOPr in several compartments of the MHb-IPN axis provided important insights regarding its physiological functions (Gardon et al., 2014). The presence of MOPr in astrocytes of the VTA, NAc and in the CA1 area of the hippocampus has also been observed using these mice (Nam et al., 2018). The MOPr-mCherry KI mouse line has also been used by Mambretti et al. (2016) to analyze the expression, localization, and potential functions of MOPr along nociceptive axons under physiological conditions. Through combination of immunostaining experiments and immunoelectron microscopy, the study showed that the receptor is present in the cytoplasm and membrane of unmyelinated axons from the sciatic nerve (Mambretti et al., 2016). While perisciatic injection of the lipophilic MOPr agonist fentanyl increased nociceptive thresholds, simultaneous co-administration with its antagonist naloxone completely reversed this physiological response, thereby confirming that axonal MOPr are functional in naïve animals (Mambretti et al., 2016).

Erbs et al. (2015) thereafter generated a double knock-in (dKI) mouse line by breeding MOPr-mCherry mice with DOPreGFP (enhanced Green Fluorescent Protein) mice (Figure 1D). In this elegant and original study, the authors have mapped the distribution of both opioid receptors throughout the nervous system and provided the results as an interactive database allowing the visualization of DOPr and MOPr at a subcellular resolution (Erbs et al., 2015). Although both receptors shared a similar distribution in some brain regions (e.g., basal ganglia), they appeared to be differentially expressed in several others
(Erbs et al., 2015). While the DOPr-eGFP showed a high signal density in the external plexiform layer of the olfactory bulb, the anterior part of the basolateral amygdaloid nucleus, the cerebral cortex and parts of the brainstem (mainly the nucleus of origin of efferents of the vestibular nerve, reticulotegmental nucleus of the pons, pontine and external cuneate nuclei), the MOPr-mCherry seemed to be predominantly expressed in the anterior olfactory nucleus, the bed nucleus of the accessory olfactory tract, extended amygdala (intermediate part of the central amygdaloid nucleus and anterior dorsal part of the medial amygdaloid nucleus), dorsal midline and intralaminar thalamic nuclei (including the paratenial, rhomboid, xiphoid and centrolateral thalamic nuclei), hypothalamus (mainly the lateral hypothalamic area, as well as the medial parts of the medial- and supra-mammillary nuclei), epithalamus ( $\mathrm{MHb}$ and fasciculus retroflexus) and parts of the brainstem (IPN, caudal part of the dorsal raphe nucleus, external part of the lateral parabrachial nucleus and parathrochlear nucleus) (Gardon et al., 2014; Erbs et al., 2015). In the spinal cord, MOPr-mCherry was mainly expressed in the superficial layers of the dorsal horn (principally lamina II), but fluorescent signals could also be detected in somas of all layers (Erbs et al., 2015). In dorsal root ganglia (DRG), both DOPr and MOPr were present in neurons with small-, medium-, and large-diameter somata (Erbs et al., 2015). However, while DOPr seemed to be more abundant in large diameter cells, MOPr appeared to be rather primarily expressed in small diameter neurons, which is in agreement with previous immunohistochemistry experiments performed in rodents (Rau et al., 2005; Scherrer et al., 2006; Wang et al., 2010).

By co-immunoprecipitation assays carried out using hippocampal tissues harvested from these dKI-FP mice, the authors also described the observation of DOPr/MOPr heteromers (Erbs et al., 2015). A few years later, heteromerization of endogenous MOPr-mCherry and DOPr-eGFP was further investigated by Derouiche et al. (2020). In this study, cointernalization of both receptors following stimulation with either DAMGO (MOPr agonist) or deltorphin II (DOPr agonist), but not SCN80, morphine or methadone was observed (Derouiche et al., 2020). Interestingly, the fate of the presumed DOPr-MOPr heteromers following ligand exposure resulted in their sorting to the lysosomal compartments (Derouiche et al., 2020). As recently reviewed in Degrandmaison et al. (2021), the DOPr displays several particularities regarding the regulation of its trafficking, including its preferential targeting to lysosomes following agonist-induced internalization, as opposed to most GPCRs, including MOPr and KOPr, which are typically recycled efficiently to the neuronal plasma membrane (Ko et al., 1999; Tsao and von Zastrow, 2000; Whistler et al., 2002; Tanowitz and von Zastrow, 2003; Tanowitz et al., 2008; Degrandmaison et al., 2021). Interestingly, this specific ligand-selective cointernalization and sorting of DOPr-MOPr heteromers to lysosomes thus suggests a DOPr-driven mechanism and might also represent a potential approach to fine-tune MOPr-mediated downstream signaling (Derouiche et al., 2020).

Grecksch et al. (2011) have investigated the role of MOPr phosphorylation on analgesic tolerance in vivo using a KI mouse expressing a mutant of the receptor in which the serine 375 is 
replaced by an alanine $\left(\operatorname{MOPr}^{S 375 A / S 375 A}\right)$ (Figure 1E). In this study, the authors first observed that treatment of mice with escalating doses of morphine for 9 days strongly upregulated MOPr expression (Grecksch et al., 2011). Interestingly, this increase was not observed in saline- or etonitazene-treated

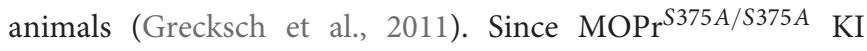
mice exhibited greater dose-dependent acute antinociceptive responses to morphine and fentanyl, phosphorylation of S375 was thus proposed to be involved in acute MOPr desensitization (Grecksch et al., 2011). These animals nevertheless still developed acute and chronic tolerance to morphine (Grecksch et al., 2011). Additional results obtained suggested that DAMGO- or etonitazene-induced tolerance required S375 phosphorylation, whereas morphine-mediated development of tolerance was S375 phosphorylation-independent (Grecksch et al., 2011).

More recently, a $\mathrm{N}$-terminally HA epitope-tagged MOPr KI mouse line (HA-MOPr) has been developed by the same group using the clustered regulatory interspaced short palindromic repeats and its associated protein-9 (CRISPR/Cas9) technology (Figure 1F; Fritzwanker et al., 2021). Relying on small guide RNAs that direct the RNA-guided nuclease Cas9, this system enables the possibility of generating $\mathrm{KO}$ and $\mathrm{KI}$ mutant mice using a relatively fast and simple methodology (Gaj et al., 2013; Hsu et al., 2014). By performing mass spectrometry (MS) analyses of immunoprecipitated HA-MOPr from brain tissues, the authors have investigated the in vivo $C$-terminal phosphorylation patterns, as well as the presence of noncanonical isoforms of the MOPr (Fritzwanker et al., 2021). While at least 12 alternative MOPr splice variants have been suggested to exist in mice, only a few studies have attempted to validate the endogenous expression of such predicted isoforms at the protein level (Pasternak and Pan, 2013; Fritzwanker et al., 2021). Given that most of the MOPr isoforms are expected to harbor $C$-terminal mutations and/or truncations, the fusion of a small epitope-tag such as HA to the $N$-terminal avoids the risk of interfering with the detection of MOPr variants. The same rationale may also be applied for the study of $C$-terminally located phosphorylation events, indicating that the HA-MOPr KI mouse line presents a valid design for the study of intracellular signaling. However, although Fritzwanker et al. (2021) have been able to identify the canonical MOPr sequence by MS on brain lysates, the authors failed to detect a significant quantity of any of the alternate $C$-terminal isoforms proposed to arise from alternative splicing. Additional assays in which the canonical HA-MOPr have been depleted from the brain lysate samples by multiple successive rounds of immunoprecipitation resulted in the same conclusion (Fritzwanker et al., 2021). Moreover, the HA-MOPr KI mouse strain has facilitated high-resolution imaging of the receptor in the mouse brain, as well as the study of the molecular mechanisms related to agonist-induced phosphorylation (Fritzwanker et al., 2021). Using phosphositespecific antibodies, the authors reported that high efficacy MOPr agonists such as methadone, fentanyl, sufentanil, and etonitazene promoted phosphorylation of $\mathrm{Ser}^{375}, \mathrm{Thr}^{370}, \mathrm{Thr}^{376}$, and $\mathrm{Thr}^{379}$ (Fritzwanker et al., 2021). Conversely, only Ser ${ }^{375}$ phosphorylation has been observed following treatment of the mice with either morphine or oxycodone, which are characterized as partial MOPr agonists (Fritzwanker et al., 2021). These results confirm previous in vitro observations reporting agonist-selective phosphorylation of the MOPr C-terminus.

Although still controversial and the current topic of heated debate, the recruitment of $\beta$-arrestin2 ( $\beta$ arr2) following morphine administration has been correlated with the deleterious side effects of opioids and reduced analgesia, suggesting that $G$ protein-biased agonists might represent an improved class of analgesics (Bohn et al., 1999; Gillis et al., 2020). To investigate the concept of biased signaling in vivo, Ehrlich et al. (2019) have therefore designed an innovative tool. In this study, a novel MOPr-Venus-YFP (yellow FP) KI mouse line was generated by homologous recombination, resulting in the expression of the MOPr-Venus-YFP replacing the native murine receptor, in order to monitor the agonist-induced differential trafficking of the receptor (Figure 1G; Ehrlich et al., 2019). The authors selected the Venus-YFP since it represents the most versatile fluorophore compatible with resonance energy transfer (RET) biosensors and the most detectable in living cells (Nagai et al., 2002; Ehrlich et al., 2019). DRG neurons from MOPr-Venus-YFP KI mice were treated with 10 different MOPr agonists, including clinically prescribed opioids, classical peptides, and $\mathrm{G} \alpha_{i / 0}$-biased agonists, to establish the specific trafficking profile induced by each compound (Table 1; Ehrlich et al., 2019). Interestingly, the authors observed that buprenorphine harbors a similar signature to the recently developed $\mathrm{G} \alpha_{i}$-biased drugs TRV 130 and PZM21 since it induced virtually no receptor redistribution or endosome translocation in vivo (Table 1) and remained largely insensitive to the overexpression of $\beta$ arr2 and G proteincoupled receptor kinase 2 (GRK2) in the assays performed in HEK293 cells (Ehrlich et al., 2019). Given that buprenorphine is already a clinically safe effective analgesic, these results suggest a reassessment of its potential as an alternative to current opioid therapeutics (Ehrlich et al., 2019).

Since 2019, several independent research groups have generated MOPr-Cre KI mouse lines, thus allowing the precise targeting, visualization, and manipulation of $\mathrm{MOPr}$-expressing neurons in vivo (Märtin et al., 2019; Bailly et al., 2020; Okunomiya et al., 2020; Zhang et al., 2020). In a first study carried out by Märtin et al. (2019), the authors successfully combined single-nucleus RNA sequencing (snRNAseq) of isolated Oprm1 positive striatal projection neurons to create a spatiomolecular map that led to the identification and characterization of the molecular diversity of neuron subtypes in the striatum. To do so, the authors genetically labeled the striatal neurons expressing Oprm1 through the generation of a MOPr-Cre KI mouse model (Figure 1H; Märtin et al., 2019). A T2A cleavable peptide sequence was inserted at the junction between the sequences encoding Oprm1 and the Cre recombinase, allowing the release of the enzyme following translation of the MOPr-T2A-Cre fusion protein (Figure 1H; Märtin et al., 2019). This mouse strain was thereafter crossed with a Cre-dependent Ail4 reporter mouse line. Following Cre-mediated recombination, the resulting mice express robust tandem dimer Tomato (tdTomato) fluorescence in Oprm1 positive striatal neurons allowing their visualization (Märtin et al., 2019). The spatiomolecular map provided by 
Märtin et al. (2019) established the differential signature of neuron subtypes and spatial markers for the identification of striatal subregions.

Designed by Okunomiya et al. (2020), a first inducible MOPr-CreER KI mouse strain has been developed by fusing the sequence encoding the Cre recombinase-modified estrogen receptor (CreER) with the T2A-like peptide sequence (Figure 1I). The resulting fusion was used to replace the STOP codon in the exon 4 of the Oprm1 gene (Okunomiya et al., 2020). Using the estrogen receptor as a regulator of the Cre-recombinase protein allow its regulation through treatments with tamoxifen (Tm), thus creating an inducible genetic system (Okunomiya et al., 2020). The Tm-dependent expression of MOPr throughout the nervous system was mapped by breeding MOPr-CreER mice with the ROSA26 reporter mice, which conditionally express a HA-tagged bright monomeric teal fluorescent protein (mTFP1) following Cre-mediated recombination (Okunomiya et al., 2020). The authors observed that Tm-inducible MOPr-CreER-mediated recombination occurred in nearly all cells that would normally express the MOPr with a correlation of more than $94 \%$ between HA-mTFP1 fluorescent and Oprm1 mRNA positive cells (Okunomiya et al., 2020). In the striatum, mTFP1-expressing cells exhibited a specific predominant localization in clusters presumably corresponding to striosomes (Okunomiya et al., 2020). Previously identified as MOPr-enriched compartments by immunohistochemistry (Arvidsson et al., 1995b; Kaneko et al., 1995; Mansour et al., 1995) and opioid binding autoradiography (Pert et al., 1976), these neural structures have been associated to diverse cognitive functions including reward prediction (Yoshizawa et al., 2018) and decision-making under conflict (Friedman et al., 2015). A similar strategy relying on the local injection of a Cre-dependent adeno-associated virus (AAV) vector in the striatum of MOPr-CreER mice has also been used to further validate the expression pattern of $M O P r$ in the striosomes (Okunomiya et al., 2020). This latter approach combining viral vectors and MOPr-CreER mice thus represents a method to spatially restrict the transgene expression in MOPr-specific cell populations (Okunomiya et al., 2020). Given that the Tm-inducible MOPr-CreER mouse model allows a temporal control of the Cre-dependent recombination, such a lineage will also open the path to future studies focusing on the pre- and post-natal development of striatal compartments (Okunomiya et al., 2020).

Bailly et al. (2020) generated a KI mouse line, namely the MOPr-eGFP/Cre, in which the sequence encoding a functional eGFP/Cre recombinase fusion protein was inserted into exon 4 of Oprm1, upstream of, and in frame with, the STOP codon (Figure 1J). The generated MOPr-eGFP/Cre protein complex was expressed in the same neurons and cellular region as its wildtype homolog as transcription remained the same and localized in the studied region of the habenula (Bailly et al., 2020). In addition to the characterization of this novel mouse model, the authors demonstrated the possibility to manipulate specific MOPr-related circuitry by combining the MOPr-eGFP/Cre mouse line with optogenetics. Indeed, the precise activation of neurons in the VTA of MOPr-eGFP/Cre mice triggered place avoidance, supporting previous results described by Tan et al. (2012), Bailly et al. (2020). The use of optogenetics has also been applied to the study of chronic pain, as recently reported by Wei et al. (2021). In this article, the authors shed light on the implication of the dorsal (DH), but not the ventral $(\mathrm{VH})$ hippocampus in the modulation of pain (Wei et al., 2021). More specifically, photostimulation of DH neurons for approximately $3 \mathrm{~h}$ was shown to relieve tactile allodynia in the spared nerve injury (SNI) neuropathic chronic pain model (Wei et al., 2021). Further experiments also suggested that the mechanism underlying the DH-mediated analgesic effects in SNI mice was dependent of $N$-Methyl-D-aspartate (NMDA) receptors, but also required the activation of MOPr (Wei et al., 2021). The development and use of optogenetic approaches will undoubtedly represent important tools for elucidating painrelated cellular mechanisms in vivo.

Another research group also took advantage of the Cremediated recombination system in order to selectively reexpress MOPr in distinct neuronal population (Zhang et al., 2020). Zhang et al. (2020) first generated a KO mouse line by inserting a floxed STOP cassette between exon 1 and 2 of the Oprm1 gene, thereby allowing re-expression of MOPr under the control of specific recombinases. Breeding of these mice with either Vglut2-Cre, Vgat-Cre, Lbx1-Cre, SNS-Cre or Nestin-Cre mice thus allowed the specific re-expression of MOPr in glutamatergic neurons, GABAergic neurons, dorsal spinal cord, small DRG neurons or in the nervous system, respectively (Figure 1K; Zhang et al., 2020). Using these newly developed conditional KI mice, the authors were able to characterize the expression, distribution pattern, as well as the contribution of each MOPr positive neuronal subpopulation in the mechanisms underlying analgesia in various pain models (Zhang et al., 2020). In a model of inflammatory pain, they observed that MOPr in Vglut2 + glutamatergic, but not GABAergic neurons mediated exogenous opioid-induced analgesia, whereas MOPr in GABAergic, but not Vglut2 + were rather involved in the endogenous opioid-induced analgesia (Zhang et al., 2020). At the spinal level, the analgesic effects of morphine mainly involve MOPr expressed in spinal glutamatergic neurons, in the context of acute pain (Zhang et al., 2020).

\section{$\delta$-Opioid Receptor Knock-In Mouse Lines}

Since its simultaneous cloning by two distinct groups in 1992, the DOPr has been recognized as a promising pharmacological target for the treatment of chronic pain, as well as various psychological and mood disorders (Evans et al., 1992; Kieffer et al., 1992; Gendron et al., 2016). In addition to the painalleviating properties reported in numerous chronic pain models (e.g., inflammatory, neuropathic, diabetic, and cancer), ligandmediated activation of the DOPr has also been associated with anxiolytic, antidepressant, cardio- and neuroprotective effects (Kamei et al., 1997; Fraser et al., 2000; Brainin-Mattos et al., 2006; Holdridge and Cahill, 2007; Otis et al., 2011; He et al., 2013; Saitoh et al., 2013; Nozaki et al., 2014; Headrick et al., 2015). Most importantly, several studies have noted that its agonists produce considerably less undesired effects than most clinically 
prescribed opioids, thus rendering it an attractive alternative target to the MOPr for chronic pain management (Gallantine and Meert, 2005; Feng et al., 2006; Codd et al., 2009). However, the molecular and cellular mechanisms governing the trafficking and signaling of the DOPr are significantly distinct from most other GPCRs, including MOPr and KOPr, contributing to our limited understanding of its regulation (reviewed in Gendron et al., 2016; Degrandmaison et al., 2021). Consequently, the development of novel genetic tools is crucial in order to investigate its involvement in pathophysiological states and its potential as a therapeutic target.

In a groundbreaking study for both the opioids and GPCRs fields, Scherrer et al. (2006) designed the first opioid receptor KI mouse line by introducing the sequence encoding the eGFP into the exon 3 of the Oprd1 mouse gene, in frame with the $5^{\prime}$-end of the STOP codon (Figure 1L). At that time, only one GFP-GPCR KI model had been generated, namely the human rhodopsin-GFP KI mouse (Chan et al., 2004). By combining homologous recombination and Cre recombinase treatment, the authors created a mouse line in which the WT DOPr is constitutively replaced by a fully functional DOPr-eGFP fusion (Figure 1L; Scherrer et al., 2006). Although a slight increase in the number of receptors and maximal GTP $\gamma \mathrm{S}$ binding were observed, the potency of selective agonists such as SNC80, deltorphin II and the endogenous peptide Met-enkephalin remained unchanged in the KI mice (Table 1; Scherrer et al., 2006). Similarly, the affinity of the antagonist $\left[{ }^{3} \mathrm{H}\right]$-naltrindole for the DOPr-eGFP was comparable to the WT receptor (Scherrer et al., 2006). While neuroanatomical analyses identified the detailed distribution of DOPr throughout the nervous system, real-time imaging in primary neurons and in vivo internalization assays have allowed the visualization of agonist-induced endocytocis of the eGFPfused DOPr (Table 1; Scherrer et al., 2006). Altogether, these results indicate that the DOPr-eGFP mouse line represents a valid and useful tool to study the receptor localization and functions in vivo.

Since its generation, the DOPr-eGFP KI mouse model has been at the core center of numerous studies. Given the fluorescent property of this mouse strain, the distribution of the DOPr throughout the central nervous system and other discrete tissues has been obviously extensively investigated (Erbs et al., 2012, 2015; Rezaï et al., 2012; Guerrero-Alba et al., 2018). These mice have also proved useful to confirm previous in vitro results indicating that the DOPr is intracellularly sequestrated and subsequently targeted to lysosomal compartments following SNC80-induced internalization (Ko et al., 1999; Tsao and von Zastrow, 2000; Whistler et al., 2002; Wang et al., 2003; Pradhan et al., 2009, 2010). The studies carried out by Pradhan et al. $(2009,2010)$ also nicely illustrate the differential regulation of SNC80- or ARM390-mediated DOPr trafficking in a liganddependent manner. Although both compounds share similar binding, G-protein activation and analgesic properties, ARM390 did not trigger internalization of the receptors, as opposed to the rapid internalization induced by SNC80 (Pradhan et al., 2010). Such cellular findings are important since they explain behavioral observations denoting that the DOPr is unable to elicit antinociceptive responses to a second dose of the agonist
SNC80, whereas a second injection of ARM390 produced potent antihyperalgesia, both 12 and $24 \mathrm{~h}$ after the first dose (Pradhan et al., 2009, 2010).

The modulation of the DOPr subcellular trafficking by various treatments and behavioral assays have also been widely investigated. For example, Dripps et al. (2020) recently described a significant increase in DOPr-eGFP expression in several forebrain regions, including the trigeminal nucleus caudalis and the trigeminal ganglia, following nitroglycerin-induced chronic migraine. Moreover, Bertran-Gonzalez et al. (2013) observed a learning-related translocation of DOPr-eGFP to the membrane of cholinergic interneurons of the NAc shell. These abovementioned studies support the importance of the pioneering DOPr-eGFP KI mouse model as a tool to investigate this receptor in vivo.

More recently, two additional DOPr KI mouse lines have been generated, both relying on epitope-tagged receptors ( $\mathrm{Su}$ et al., 2017; Degrandmaison et al., 2020). The first model, generated by $\mathrm{Su}$ et al. (2017), has been developed using the Transcription Activator-Like Effector Nucleases (TALEN) system. This approach allowing a one-step recombination uses a sequence-specific DNA-binding domain fused to a non-specific FokI nuclease domain. Following binding to the predetermined DNA sequence, the enzyme will induce a double strand DNA break resulting in the engagement of DNA repair mechanisms, which in turn facilitates homologous recombination and the proper insertion of specific sequences within the targeted regions (Gaj et al., 2013; Joung and Sander, 2013; Su et al., 2017). In their study, the authors generated a mouse line expressing a $N$-terminally HA-tagged DOPr replacing the endogenous receptor (Figure 1M; Su et al., 2017). These mice were used to investigate the expression and distribution of the HA-DOPr in various brain sections and in the spinal cord (Su et al., 2017). The genetic design of this unique mouse strain also allows for the deletion of the receptor in specific tissues and/or cells following the removal of the floxed HA-DOPr sequence by a Cre recombinase (Su et al., 2017). This possibility has been further investigated by breeding HA-DOPr mice with Nestin-Cre mice in order to generate mice with a neural-specific DOPr KO ( $\mathrm{Su}$ et al., 2017). These mouse lines represent powerful tools to study the molecular, cellular, and physiological functions of DOPr in distinct cell populations.

Using classical homologous recombination, our group has recently generated FLAG-DOPr KI mice (Degrandmaison et al., 2020). To create this lineage, we first designed a FLAG-DOPr$\mathrm{KO}$ mouse strain containing the sequence encoding the FLAG epitope at the $5^{\prime}$-end of the Oprd1 mouse gene (immediately after the START codon) and a translational STOP cassette flanked by two loxP sites inserted within the $5^{\prime}$-untranslated region $\left(5^{\prime}\right.$ UTR) (Degrandmaison et al., 2020). The presence of the STOP cassette disables the expression of FLAG-DOPr in all tissues, and by doing so, confers initial genotypic characteristics of a KO model (Degrandmaison et al., 2020). The breeding of these FLAG-DOPr KO mice with Zp3-Cre mice resulted in the excision of the floxed sequence which enables the expression of FLAGtagged DOPr in all tissues that would normally express the WT DOPr (Figure 1N; Degrandmaison et al., 2020). An important 
feature of the FLAG-DOPr KO model resides in the possibility of generating conditional KI using distinct Cre recombinases allowing to rescue DOPr expression in a tissue- and/or cellspecific manner (discussed in section "Epitope-tagged G proteincoupled receptors knock-in mouse lines") (Degrandmaison et al., 2020). The expression levels and distribution in the brain, as well as the functional characterization (i.e., G protein coupling, locomotor and pain behavioral studies) of the endogenous FLAG-DOPr demonstrated that FLAG-DOPr KI mice display similar behavioral and pharmacological properties as WT DOPr mice (Table 1; Degrandmaison et al., 2020). We used the unique properties of the FLAG-DOPr KI mice to reveal the first in vivo interactome of a GPCR (Degrandmaison et al., 2020). Indeed, proteomic analyses of the immunoprecipitated FLAG-DOPr from the forebrain of FLAG-DOPr KI mice allowed the identification of previously reported and, most importantly, novel endogenous DOPr-interacting proteins (Degrandmaison et al., 2020). Among the newly identified interactors, Rab10 has been further characterized and shown to be involved in the cellsurface targeting of the DOPr (Degrandmaison et al., 2020). Such an approach opened the path to exciting perspectives regarding the investigation of protein-protein interactions in vivo (further discussed in section "Epitope-tagged G protein-coupled receptors knock-in mouse lines").

\section{к-Opioid Receptor Knock-In Mouse Lines}

As for the other OPr subtypes, activation of the KOPr has also been reported to elicit analgesia (Pathan and Williams, 2012). However, the wide range of adverse effects associated with its agonists, including dysphoria, aversion, stress-induced anxiety, sedation and psychotomimesis, has limited their clinical development for chronic pain-related treatment (Pfeiffer et al., 1986; Dykstra et al., 1987; Roth et al., 2002; Land et al., 2009). Actual research thus explores various strategies to limit these undesired physiological responses by focusing on the design of peripherally restricted KOPr agonists, $G$ protein-biased agonists or drugs targeting several OPrs simultaneously (Paton et al., 2020). Nevertheless, the selective KOPr antagonist JNJ67953964 or Opra Kappa (previously LY2456302) is currently under clinical development for the treatment of major depressive disorders (Reed et al., 2018; Jacobson et al., 2019). KOPrtargeted therapeutics also represent promising avenues for the management of other pathologies such as schizophrenia and drug addiction (Clark and Abi-Dargham, 2019). Although the first $\mathrm{KOPr} \mathrm{KO}$ animal has been generated more than two decades ago, only two KI mouse lines, namely the KOPr-Cre and KOPr-tdT, have been developed until now (Cai et al., 2016; Chen et al., 2020). Considering the significant therapeutic potential of KOPr in numerous pathophysiological conditions, further research efforts focusing on this receptor are expected in the coming years.

Cai et al. (2016) generated a KOPr-Cre KI mouse line, representing the first developed OPr-Cre recombinase mouse strain. In these animals, the coding region of the Oprk1 gene in exon 2 is replaced with the sequence encoding a Cre Recombinase (Figure 1O; Cai et al., 2016). Such a model allows for the conditional manipulation of cells specifically expressing $\mathrm{KOPr}$ (Cai et al., 2016). The authors further characterized the Cremediated recombination by breeding KOPr-Cre KI mice with mice displaying a Cre-dependent allele Rosa ${ }^{\text {IsltdTomato }}$ (Cai et al., 2016). Results indicated that KOPr is expressed in numerous cell types throughout the organism, including the cerebral cortex, NAc, DRG, striatum, heart, lung, and liver (Cai et al., 2016).

More recently, Chen et al. (2020) published three-dimensional (3D) images of the brain distribution of KOPr, a first for a GPCR, using a newly generated mouse line expressing the receptor fused to the fluorescent protein tdTomato (tdT) at its $C$-terminal extremity (Figure 1P). This novel mouse strain, henceforth referred to as KOPr-tdT, has been used to investigate the agonistinduced internalization of $\mathrm{KOPr}$, as well as its neuroanatomical and cellular distribution (Table 1; Chen et al., 2020). To do so, the authors adapted the electrophoretic tissue clearing (ETC)CLARITY method previously described by Kim et al. (2015) to generate 3-D images of KOPr brain distribution (Chen et al., 2020). The (ETC)-CLARITY clearing tissue approach, involving perfusion of animals with fixatives and acrylamidebased hydrogel and removal of lipids by detergents, allows the obtention of an optically transparent tissue while conserving its structural integrity (Kim et al., 2015; Chen et al., 2020). The elegantly displayed 3-D videos and brain images presented by Chen et al. (2020) suggest that KOPr is expressed in regions related to pain modulation, reward and aversion, as well as other areas for which a clear role has not yet been established such as the claustrum, dorsal endopiriform nucleus, lateral habenula and paraventricular nucleus of the thalamus. Although the specific structures were not identified, modest expression levels of KOPr were also observed in the spinal cord and DRGs (Chen et al., 2020). The optimized (ETC)-CLARITY tissue clearing method and the novel KOPr-tdT mouse line developed by the authors therefore represent useful tools for future investigations regarding the neuroanatomy of GPCRs, including $\mathrm{OPr}$, as well as KOPr functions in various circuitries.

\section{Nociceptin/Orphanin FQ Receptor Knock-In Mouse Lines}

Officially classified as a non-opioid member of the OPr family, the NOPr displays a unique pharmacological profile characterized by a low affinity for standard opioid peptides and antagonists, including the clinically approved drug naloxone (Pathan and Williams, 2012; Borsodi et al., 2019). Moreover, despite exhibiting $55-59 \%$ of sequence identity with the other three OPr subtypes, the NOPr is associated with dichotomous physiological effects regarding the modulation of pain transmission (Reinscheid et al., 1995; Grisel et al., 1996; Mogil et al., 1996a,b; Xu et al., 1996; Yamamoto et al., 1997; Mogil and Pasternak, 2001; Degrandmaison et al., 2021). Depending on several factors such as the dose, route of administration, type of pain stimulus, species or strains, agonists of the NOPr have been shown to induce both pro-nociceptive and analgesic actions (Reinscheid et al., 1995; Grisel et al., 1996; Mogil et al., 1996a,b; Xu et al., 1996; Yamamoto et al., 1997; Mogil and Pasternak, 2001; Donica et al., 2013). For example, while the spinal administration of its endogenous ligand 
nociceptin/orphanin FQ (N/OFQ) is mainly antinociceptive, intracerebroventricularly injected N/OFQ was found to block opioid-mediated analgesia, a physiological response frequently referred to as "anti-opioid activity" (Reinscheid et al., 1995; Grisel et al., 1996; Mogil et al., 1996a,b; Xu et al., 1996; Yamamoto et al., 1997; Mogil and Pasternak, 2001). These observations highlight the distinctively complex regulation of the NOPr and its close connection to the classical opioid system. As a result of its later discovery, the NOPr is the least well-characterized receptor as compared to the other OPr subtypes (Bunzow et al., 1994; Fukuda et al., 1994; Mollereau et al., 1994; Wang et al., 1994). Hence, the development of novel genetic tools is crucial to further improve our knowledge of the molecular and cellular mechanisms regulating this receptor, as well as its involvement in pain-, memory-, stress/anxiety- and drug rewardrelated pathways.

To our knowledge, the NOPr-eGFP and NOPr-eYFP KI mouse lines represent the only available KI animal models for this receptor (Ozawa et al., 2015; Mann et al., 2019). In both cases, using a similar approach as described for the generation of DOPr-eGFP and MOPr-mCherry (see sections " $\mu$-opioid receptor knock-in mouse lines" and " $\delta$-opioid receptor knockin mouse lines"), the authors specifically inserted the sequence encoding the eGFP or eYFP into the Oprl1 mouse gene, in frame and $5^{\prime}$ from the STOP codon, resulting in mouse strains expressing functional NOPr-FP $C$-terminal fusions replacing the native NOPr (Figures 1Q,R; Ozawa et al., 2015; Mann et al., 2019). While the NOPr-eGFP has been mainly used to study the in vivo distribution and agonist-induced phosphorylation of the receptor, primary culture of ventral midbrain neurons from NOPr-eYFP KI mice have been employed to visualize its internalization (Table 1; Ozawa et al., 2015; Mann et al., 2019). In a first study, Ozawa et al. (2015) have mapped the expression of the NOPr-eGFP in the spinal cord, DRG neurons and numerous brain regions involved in both pain (e.g., thalamus, MHb, vlPAG and locus coeruleus) and reward (e.g., NAc, VTA, MHb, amygdala, hippocampus and IPN), which supports a role for this receptor in such circuitries. While previous in vitro $\left[{ }^{3} \mathrm{H}\right]-\mathrm{N} / \mathrm{OFQ}$ radiography performed by Neal et al. (1999) in DRGs resulted in no binding, the presence of NOPr-eGFP in these cells is in agreement with in situ hybridization and electrophysiological experiments (Murali et al., 2012; Ozawa et al., 2015). Expression of NOPr in the spinal cord and DRG neurons is also consistent with the effects of intrathecally administered N/OFQ on nociception (Xu et al., 1996; Yamamoto et al., 1997; Ozawa et al., 2015). Given that supraspinal administration of N/OFQ can reverse the morphineinduced antinociceptive responses, it is interesting to note that the authors have identified cells co-expressing both NOPr and MOPr in various brain regions, as well as DRG neurons (Grisel et al., 1996; Ozawa et al., 2015). Further studies will however be needed to provide new insights on the complex interplay between these receptors.

In a following study, the authors analyzed the spinal distribution of NOPr in a chronic neuropathic pain model of spinal nerve ligation (SNL) (Ozawa et al., 2018). The results indicated that a decrease in the NOPr-eGFP fluorescent signal was observed in the spinal dorsal lamina I and II outer, regions recognized to mediate noxious heat stimuli, as well as in the L4 and L5 ipsilateral DRGs of mice that had undergone SNL (Ozawa et al., 2018). The capacity to visualize the NOPr-eGFP in discrete locations following the induction of pathophysiological states or treatments further reinforces the usefulness of FP-fused receptor KI mouse lines.

More recently, Mann et al. (2019) have investigated the differential agonist-induced NOPr phosphorylation in brains of NOPr-eGFP mice. Using newly developed phosphosite-specific antibodies and mass spectrometry analyses, the authors have identified the mouse residues Ser ${ }^{343}, \mathrm{Ser}^{348}$, $\mathrm{Thr}^{359}$, and $\mathrm{Ser}^{360}$ of the NOPr as phosphorylated in vivo following the intraperitoneal administration of the non-peptide full agonist AT-202 (Mann et al., 2019). Conversely, $\operatorname{Ser}^{346}$ and $\operatorname{Ser}^{351}$ appeared to be constitutively phosphorylated in the absence of a ligand, but an increase of phosphorylation was nevertheless observed following agonist treatment (Mann et al., 2019). The role of GRK2 and GRK3 in the AT-202-induced NOPr phosphorylation was also validated in vivo using compound 101, a selective GRK2/3 inhibitor (Mann et al., 2019). Most importantly, ligand-specific patterns of agonist-mediated phosphorylation and internalization have also been investigated (Mann et al., 2019). While NOPr agonists AT-202, Ro64-6198 and SCH221510 induced a significant internalization and phosphorylation at $\mathrm{Ser}^{343}, \mathrm{Ser}^{348}$, $\mathrm{Thr}^{359}$, and $\mathrm{Ser}^{360}$, receptors of the ventral midbrain neurons isolated from NOPr-eYFP mice exhibited only a slight internalization following stimulation with $\mathrm{NNC}$ 63-0532 (Table 1; Mann et al., 2019). On the other hand, MCOPPB administration induced strong NOPr internalization and phosphorylation at $\mathrm{Ser}^{343}$, but only a weak phosphorylation signal was detectable at $\mathrm{Ser}^{348}$, $\mathrm{Thr}^{359}$, and $\mathrm{Ser}^{360}$ (Mann et al., 2019). The complex pharmacology and various physiological responses attributed to the NOPr might therefore be explained by such agonist-selective differential phosphorylation, which would presumably influence subsequent signaling.

\section{Limitations, Perspectives and Future Directions}

The arsenal of currently developed OPr KI mouse lines provides exciting perspectives regarding future studies investigating the molecular and cellular mechanisms underlying opioid physiology and associated disorders. As discussed below, the considerable number of available OPr KI mice should allow for the rapid development of additional unique models that could be pivotal for both the opioids and the GPCRs fields.

\section{Knock-In Mouse Lines Harboring a G Protein-Coupled Receptors-Fluorescent Protein Fusion}

Breeding of already existing KI mouse lines could represent a relatively simple strategy to investigate the complex interplay between OPr subtypes. As describe above, such an approach has already been used for the generation of DOPr-eGFP/MOPrmCherry dKI mice (Figure 1D; Erbs et al., 2015). Similarly, the interplay between $\mathrm{KOPr} / \mathrm{DOPr}$ or $\mathrm{KOPr} / \mathrm{NOPr}$ could be 
investigated through the development of DOPr-eGFP/KOPr$\mathrm{tdT} \mathrm{dKI}$ and NOPr-eGFP/KOPr-tdT dKI mouse lineages, respectively. Furthermore, breeding of the existing MOPrmCherry/DOPr-eGFP dKI mouse with a KI mouse harboring a KOPr fused to a complementary FP (e.g., mTagBFP2) could result in an interesting and unique triple KI model allowing for the simultaneous mapping of all three OPr subtypes (Kleeman et al., 2018). A plethora of possibilities also emerged when considering other KI mouse lines outside of the OPr family, as exemplified by the generation of a MOPr-mCherry/CX3CR1-eGFP dKI reporter mouse line, a model used to investigate microglial expression of MOPr in the brain and spine (Maduna et al., 2019).

Although KI mice designed with a GPCR-FP fusion have been and are still considerably useful to investigate many aspects of the receptor's physiology, these lineages seem to be less appropriate for some particular applications. The main concern of these strains relies on the significant size of the fused FP (>25 kDa), which might be problematic for some analyses. For example, despite the undeniable advances provided by the DOPr-eGFP KI mouse model, the fusion of the FP to the $C$-terminal of the DOPr has raised controversy regarding its subcellular localization (Table 1; reviewed in Gendron et al., 2016). While a predominant intracellular localization of the DOPr under basal conditions has been reported in numerous studies (Pasquini et al., 1992; Arvidsson et al., 1995a; Cahill et al., 2001a,b; Wang and Pickel, 2001; Lucido et al., 2005; Gendron et al., 2006; Shiwarski et al., 2017a,b), high membrane expression of DOPr-eGFP has been described in tissues from KI mice (Table 1; Scherrer et al., 2006). Although Scherrer et al. (2006) confirmed that the DOPreGFP-induced signaling was similar to the WT DOPr in transfected HEK293 cells, another study reported that the addition of a GFP to either the $N$ - or $C$-terminus of the DOPr significantly altered its subcellular distribution in PC12 cells (Wang et al., 2008). Ozawa et al. (2015) also noticed a progressive increase in the number of plasma membranelocalized receptors in WT, heterozygous, and homozygous NOPr-eGFP KI mice, respectively (Table 1). Alteration of the trafficking of other GPCRs, including the cannabinoid 1 receptor, muscarinic M4 receptor and $\beta$-adrenergic receptors, has also been described following the addition of an eGFP (McLean and Milligan, 2000; Madziva and Michael, 2001; McDonald et al., 2007). Altogether, these results suggest that the $N$-terminal fusion of an eGFP to a receptor might facilitate its maturation and transport along the biosynthetic pathway or perhaps increase its stability. Conversely, Wang et al. (2008) observed that the DOPr plasma membrane density remained unchanged following fusion of short epitope-tag sequences (e.g., $\mathrm{HA}$ or $\mathrm{Myc}$ ) to its $\mathrm{N}$-terminal extremity, indicating that the use of smaller tags might represent a more suitable strategy depending on the experiments to be performed. It is worth noting that the cell-surface targeting of the DOPr-eGFP in KI mice could still be increased under specific physiological conditions, in agreement with previous observations (Bertran-Gonzalez et al., 2013). Current progress in the development of smaller FPs, such as the miRFP670nano $(\sim 17 \mathrm{kDa})$, could eventually represent appealing alternatives for the development of future tools allowing in vivo imaging of GPCRs (Oliinyk et al., 2019).

For specific experimental purposes, the generation of GPCRFP KI mouse lines for in vivo fluorescence imaging might be ultimately replaced by newly developed technologies. For example, Esteoulle et al. (2020) have recently synthesized a bright fluorogenic near-infrared probe enabling the specific and background-free imaging of an endogenous GPCR in living mice. Absorption and emission of the dimeric probe in the near-infrared region optical window permits optimal in vivo imaging by minimizing the light scattering and absorption in blood and tissues, resulting in enhanced tissue penetration (Stolik et al., 2000; Hilderbrand and Weissleder, 2010; Yuan et al., 2013; Esteoulle et al., 2020). Using the oxytocin receptor as a prototype to develop this unprecedented whole animal fluorescence imaging method, the authors successfully labeled endogenous receptors, an achievement once considered as excessively challenging due to the low expression levels of GPCRs (Esteoulle et al., 2020). Given that this first-of-kind approach could presumably be transposed to other GPCRs, the use of such probes might eventually be applied for the study of OPr. Similarly, although it was not carried out in living animals, advances have also been recently denoted for the visualization of endogenous MOPr and DOPr in striatal cholinergic interneurons (Arttamangkul et al., 2021). Using NAI-A594, a ligand-directed labeling agent, the authors fluorescently labeled both OPr from live brain slices and concluded that MOPr and DOPr function independently, despite being localized in the same neurons (Arttamangkul et al., 2021).

\section{Epitope-Tagged G Protein-Coupled Receptors Knock-In Mouse Lines}

In some contexts, endogenously expressed epitope-tagged GPCRs represent an interesting alternative to a GPCR-FP fusion. As mentioned previously, peptide epitopes such as HA, FLAG or Myc might reduce the probability of altering the trafficking and function of the targeted receptor due to their small size ranging from 8 to 10 amino acids. This approach has been used to generate the HA-DOPr, FLAG-DOPr and HA-MOPr KI mice (Su et al., 2017; Degrandmaison et al., 2020; Fritzwanker et al., 2021). Since the $C$-terminal extremity of GPCRs represents a major site for post-translational modifications (PTM) and for the binding of effector proteins, fusion of the epitope-tag sequence to the $N$-terminal of the receptor has been carried out to prevent the potential gain and/or loss of interacting partners that could be mediated by an intracellular tag (Degrandmaison et al., 2021). Combination of these newly developed mouse lines with proteomics opened the path to a plethora of possibilities regarding the study of protein-protein interactions and PTMs. Akin to other GPCRs, OPr can undergo a wide range of constitutive and dynamic PTMs including glycosylation, palmitoylation, phosphorylation, and ubiquitination (Lemos Duarte and Devi, 2020; Degrandmaison et al., 2021). Since the fusion of the HA- or FLAG-sequence to the GPCR significantly facilitates its immunoprecipitation and enrichment from tissues, PTMs regulating OPr can be investigated in vivo using proteomic analyses or specific antibodies, as carried 
out by Fritzwanker et al. (2021). We have recently revealed the endogenous interactome of the DOPr by LC-MS/MS analyses performed on immunoprecipitated FLAG-DOPr from the forebrain of KI mice, thus leading to the identification of several potential DOPr-interacting proteins (Degrandmaison et al., 2020). Comparative interactome analyses between various regions of the brain, as well as other tissues including spinal cord and DRGs, could be pivotal in elucidating distinct DOPr functions throughout the nervous system. Similarly, comparison of the in vivo DOPr interactome following specific treatments or in various pain models (e.g., chronic morphine administration, inflammatory pain model) could provide leads for the study of the molecular mechanisms governing DOPr in pain-related pathways. Moreover, the complex interplay between DOPr and MOPr could also be investigated through the generation of a dKI mouse model, this time by breeding FLAG-DOPr mice with HA-MOPr mice. A compelling feature of such strain would reside in the possibility to study the protein-protein interactions of the receptors both simultaneously and independently. Indeed, distinct DOPr-, MOPr-, and DOPr/MOPr-interacting partners could be determined using a protocol combining sequential immunoprecipitations of FLAG- and HA-associated proteins, as well as LC-MS/MS analyses. An analogous strategy could be applied for the study of the KOPr- and NOPrassociated interactomes.

Similarly, such an approach could also be employed to investigate the connections existing between $\mathrm{OPr}$ and other physiological systems. For example, co-immunoprecipitation and BRET assays have suggested that the DOPr forms heteromers with the $\alpha_{2 A}$-adrenergic receptor $\left(\alpha_{2 A}\right.$-AR) in HEK293 cells (Rios et al., 2004). Most interestingly, analgesic synergy between DOPr and $\alpha_{2 A}$-AR has been observed following the spinal co-administration of some of their respective agonists (see Chabot-Doré et al., 2015 for a review). Combination therapy, in which the co-administered $\alpha_{2 A}$-AR agonist acts as an adjuvant to opioids, represents a promising alternative with significant potential clinical impacts as it can conceivably improve the analgesic response while reducing opioid-related side effects (Chabot-Doré et al., 2015). To our knowledge, despite these biochemical and physiological observations, a direct interaction between DOPr and $\alpha_{2 A}$-AR in native tissues has not been reported yet, and very little is known about the downstream signaling mechanisms participating in the synergistic opioidadrenoreceptor axis (Chabot-Doré et al., 2015). Since Lu et al. (2009) have already generated a $N$-terminally HA-tagged $\alpha_{2 A^{-}}$ AR KI mouse line (HA- $\left.\alpha_{2 A}-\mathrm{AR}\right)$, the interplay between these two systems could be investigated through the generation of a FLAG-DOPr/HA- $\alpha_{2 A}$-AR dKI mouse.

Until now, visualization of FLAG-specific labeling in brain sections of FLAG-DOPr-KI mouse has been impaired by high fluorescence background signal (Degrandmaison et al., 2020). Non-specific binding of FLAG antibodies has been reported in brain tissues and cells by several research groups (Schäfer and Braun, 1995; Ferrando et al., 2015). In their study, Ferrando et al. (2015) generated numerous KI proteins harboring a 3xFLAG epitope that greatly improved their detection in all mice tissues except in the adult brain where non-specific labeling was still observed. An additional challenge resides in the low expression levels of DOPr, akin to other GPCRs, which certainly impacts on our capacity to visualize specific immunolabeling as compared to systems in which endogenous proteins are fundamentally highly abundant or overexpressed (e.g., using viral infections) (Degrandmaison et al., 2020). Using brain slices from a transgenic mouse strain expressing FLAG-MOPr targeted to catecholamine neurons, Arttamangkul et al. (2008) have successfully observed FLAG-specific staining of the receptors. However, one must keep in mind that the endogenous expression levels of DOPr and MOPr are different, and that the genetic approach used by the authors resulted in the expression of approximately twofold more FLAG-MOPr as compared to WT littermates, which might potentially facilitate the detection of the endogenous receptors (Arttamangkul et al., 2008). The generation of novel tools such as the FLAG-M5 monoclonal antibody (Sigma-Aldrich, Darmstadt, Germany, \#F4042), which specifically recognizes $N$-terminal Methionine-FLAG fusion protein ( $N$-term. Met-FLAG-protein), thus corresponding to the sequence harbored by the FLAGDOPr-KI mouse, might represent an interesting alternative to overcome these challenges.

Conversely, the possibility to perform high-resolution imaging using HA-specific antibodies in neurons of HAMOPr or HA-DOPr KI mice opens the path to upcoming investigations regarding their respective subcellular localization and intracellular trafficking. This approach also allows the study of the cellular redistribution of receptors in vivo in pathological conditions or following treatments. Akin to the DOPr (discussed in section " $\delta$-opioid receptor knock-in mouse lines"), changes in the expression and/or subcellular localization of the MOPr in specific physiological conditions have been previously reported. In addition to the increase in MOPr expression following a 9 days treatment with escalating doses of morphine mentioned above (see section " $\mu$-opioid receptor knock-in mouse lines”) (Grecksch et al., 2011), upregulation of MOPr has also been observed in corneal nerve fibers and trigeminal sensory neurons in a mouse model of inflammatory corneal pain (Joubert et al., 2020). In this study, topical ocular administration of DAMGO resulted in corneal hypersensitivity relief associated with inflammatory ocular pain (Joubert et al., 2020). On the other hand, downregulation of MOPr gene and protein expression has been described in L3-L5 DRGs removed from the ipsilateral side of rats with chronic inflammation of the knee joint, as well as in the synovium of adjuvant-induced monoarthritic rat knee joints (McDougall et al., 2004; Li et al., 2005). As another example, the fractionation and immunofluorescence assays performed by Mousa et al. (2013) revealed that MOPr displayed a predominant intracellular localization, and extensive co-localization with Rab7 in lysosome-associated membrane glycoprotein-1 (LAMP-I) positive perinuclear lysosomal compartments in DRG neurons of diabetic rats. By interfering with the Rab7-mediated lysosomal targeting of the receptors using intrathecally injected Rab7siRNAs, the authors successfully restored the plasma membrane density of MOPr, as well as opioid responsiveness toward better pain relief in an animal model of diabetic neuropathic pain (Mousa et al., 2013). 
Furthermore, the genetic design used to create the abovementioned FLAG-DOPr KO mouse line (see section " $\delta$-opioid receptor knock-in mouse lines") enables the generation of conditional KI animals (Degrandmaison et al., 2020). Indeed, the specific Cre-driven excision of the translational STOP cassette can allow the rescue of DOPr expression in target tissue and/or cells. As a proof of concept, we used the recombinant adenoassociated virus rAAV2/9-CBA-Cre-GFP (Abdallah et al., 2018), which predominantly targets lumbar DRGs following intrathecal administration, to specifically re-express the receptor in these neurons (Degrandmaison et al., 2020). In a CFA-induced chronic pain model, our results indicated that the antihyperalgesic effects of deltorphin II, a DOPr specific agonist, were partially reinstated 6 weeks post viral infection, thereby supporting a role for DOPr localized on primary afferents in the control of pain induced by a thermal stimulus (Degrandmaison et al., 2020). The FLAGDOPr-KO mouse therefore represents a powerful genetic tool to decipher the roles of the DOPr in specific targeted regions.

\section{Future Directions}

The constant progress in the field of genome editing led to the development of numerous revolutionary tools including the CRISPR/Cas9 technology. As already described for the generation of the HA-MOPr KI mouse line (see section " $\mu$ opioid receptor knock-in mouse lines"), this approach enables the possibility of generating $\mathrm{KO}$ and $\mathrm{KI}$ mutant mice by deleting, inserting or modifying a specific targeted gene (Hsu et al., 2014; Hall et al., 2018). In an attempt to guide future pharmacological treatments toward precision medicine, a tool such as CRISPR/Cas9 will undoubtedly potentiate the generation of novel mouse lines harboring mutations representative of identified SNPs, as described above for the MOPr-A118G, or other relevant mutations that would better our understanding of the molecular mechanisms related to opioid physiology (Mague et al., 2009). Possible involvement of the DOPrF27C, KOPr-D374N or additional MOPr variants in different pathophysiological conditions could also be studied in vivo using the CRISPR/Cas9 technology (Gelernter and Kranzler, 2000). Moreover, as discussed for the MOPr-Cre strain (see section " $\mu$-opioid receptor knock-in mouse lines"), combination of KI mouse lines with other emerging cutting-edge technologies, such as opto- and chemo-genetics, is expected to play a significant role in providing new insights on opioid physiology (Gillis et al., 2020). Members of the OPr family have been established once again as leading-edge receptors for the development of novel tools, as demonstrated by the recent design of the lightactivable MOPr chimera "Opto-MOR" and the unique KOPrDREADD (Designer Receptor Exclusively Activated by Designer Drug) chemogenetic system allowing the in vivo modulation of neuronal activity (Siuda et al., 2015; Vardy et al., 2015).

Recent advances in the GPCR field have also contributed to the improvement and development of novel technologies enabling a better understanding of their complex physiology. After several years of challenging optimization, the use of Resonance Energy Transfer (RET)-based biosensors in living organisms has finally been successful (van Unen et al., 2015; Kono et al., 2017). In their study, Kono et al. (2017) described the generation of a genetically engineered mouse used for the in vivo real-time imaging of the sphingosine-1-phosphate receptor 1 $\left(\mathrm{SIP}_{1}\right)$ signaling by reporting the interaction with $\beta$ arr2. Upon receptor activation, complementation of the engineered firefly split luciferase fragments produces an active enzyme complex that, in the presence of ATP and luciferin, generates light detectable by bioluminescence imaging (Kono et al., 2017). If transposed to the study of OPr, such bioluminescent mouse models might have a significant impact on the development of novel opioid therapeutics, as well as on our understanding of the specific downstream signaling pathways activated by distinct agonists.

\section{CONCLUSION}

Given their substantial therapeutic importance, OPr have been widely used as prototypic GPCRs for the development of several novel in vivo genetic tools. Indeed, OPr KI mouse lines have been the basis of important advances in the GPCR field, including the generation of the second GFP-GPCR fusion KI mouse model (Scherrer et al., 2006), the first 3-D brain images to investigate the endogenous distribution of a GPCR (Chen et al., 2020), and the first in vivo interactome of a GPCR (Degrandmaison et al., 2020). The combination of the recently developed OPr-Cre KI mice, as well as the "KOPr-DREADD" and the light-activable "Opto-MOR" with opto- and chemo-genetic approaches opens the path to the visualization and, more importantly, the direct in vivo manipulation of specific neurons and targeted circuitries (Siuda et al., 2015; Vardy et al., 2015; Cai et al., 2016; Märtin et al., 2019; Bailly et al., 2020; Okunomiya et al., 2020). In addition to the above-mentioned perspectives, the myriad of exciting possibilities regarding the generation of unique mouse strains is further multiplied when considering the breeding of KI and KO models. Finally, the approaches developed to investigate the functions of OPr in vivo could also be pivotal for the study of other GPCRs, as the genetic strategies can be conceivably transposed.

\section{AUTHOR CONTRIBUTIONS}

JD, SR-H, J-LP, and LG wrote the manuscript. All the authors contributed to the article and approved the submitted version.

\section{FUNDING}

This work was supported by a grant from the Canadian Institutes of Health Research (to J-LP and LG, \#PJT-162103). JD received a Ph.D. scholarship from the Fonds de Recherche du QuébecSanté (FRQ-S).

\section{ACKNOWLEDGMENTS}

Figure was created using BioRender.com. 


\section{REFERENCES}

Abdallah, K., Nadeau, F., Bergeron, F., Blouin, S., Blais, V., Bradbury, K. M., et al. (2018). Adeno-associated virus $2 / 9$ delivery of Cre recombinase in mouse primary afferents. Sci. Rep. 8:7321. doi: 10.1038/s41598-018-25626-y

Anton, R. F., Oroszi, G., O’Malley, S., Couper, D., Swift, R., Pettinati, H., et al. (2008). An evaluation of $\mu$-opioid receptor (OPRM1) as a predictor of naltrexone response in the treatment of alcohol dependence: results from the combined pharmacotherapies and behavioral interventions for alcohol dependence (COMBINE) study. Arch. Gen. Psychiatry 65, 135-144. doi: 10. 1001/archpsyc.65.2.135

Arttamangkul, S., Platt, E. J., Carroll, J., and Farrens, D. (2021). Functional independence of endogenous $\mu$ - and $\delta$-opioid receptors co-expressed in cholinergic interneurons. eLife 10:e69740. doi: 10.7554/eLife.69740

Arttamangkul, S., Quillinan, N., Low, M. J., von Zastrow, M., Pintar, J., and Williams, J. T. (2008). Differential activation and trafficking of $\mu$-opioid receptors in brain slices. Mol. Pharmacol. 74, 972-979. doi: 10.1124/mol.108. 048512

Arvidsson, U., Riedl, M., Chakrabarti, S., Lee, J., Nakano, A., Dado, R., et al. (1995b). Distribution and targeting of a mu-opioid receptor (MOR1) in brain and spinal cord. J. Neurosci. 15, 3328-3341. doi: 10.1523/JNEUROSCI.15-0503328.1995

Arvidsson, U., Dado, R. J., Riedl, M., Lee, J. H., Law, P. Y., Loh, H. H., et al. (1995a). $\delta$-Opioid receptor immunoreactivity: distribution in brainstem and spinal cord, and relationship to biogenic amines and enkephalin. J. Neurosci. 15, 1215-1235. doi: 10.1523/JNEUROSCI.15-02-01215.1995

Bailly, J., Del Rossi, N., Runtz, L., Li, J. J., Park, D., Scherrer, G., et al. (2020). Targeting morphine-responsive neurons: generation of a knock-in mouse line expressing cre recombinase from the Mu-opioid receptor gene locus. eNeuro 7:ENEURO.0433-19.2020. doi: 10.1523/ENEURO.0433-19.2020

Bertran-Gonzalez, J., Laurent, V., Chieng, B. C., Christie, M. J., and Balleine, B. W. (2013). Learning-related translocation of $\delta$-Opioid receptors on ventral striatal cholinergic interneurons mediates choice between goal-directed actions. J. Neurosci. 33, 16060-16071. doi: 10.1523/JNEUROSCI.1927-13.2013

Bohn, L. M., Lefkowitz, R. J., Gainetdinov, R. R., Peppel, K., Caron, M. G., and Lin, F. T. (1999). Enhanced morphine analgesia in mice lacking beta-arrestin 2. Science 286, 2495-2498. doi: 10.1126/science.286.5449.2495

Borsodi, A., Bruchas, M., Caló, G., Chavkin, C., Christie, M. J., Civelli, O., et al. (2019). Opioid receptors (version 2019.4) in the IUPHAR/BPS Guide to Pharmacology Database. IUPHAR/BPS Guide Pharmacol. CITE 2019. doi: 10.2218/gtopdb/F50/2019.4

Brainin-Mattos, J., Smith, N. D., Malkmus, S., Rew, Y., Goodman, M., Taulane, J., et al. (2006). Cancer-related bone pain is attenuated by a systemically available $\delta$-opioid receptor agonist. Pain 122, 174-181. doi: 10.1016/j.pain.2006.01.032

Brooks, J., and Tracey, I. (2005). From nociception to pain perception: imaging the spinal and supraspinal pathways. J. Anat. 207, 19-33. doi: 10.1111/j.1469-7580. 2005.00428.x

Bunzow, J. R., Saez, C., Mortrud, M., Bouvier, C., Williams, J. T., Low, M., et al. (1994). Molecular cloning and tissue distribution of a putative member of the rat opioid receptor gene family that is not a mu, delta or kappa opioid receptor type. FEBS Lett. 347, 284-288. doi: 10.1016/0014-5793(94)00561-3

Cahill, C. M., McClellan, K. A., Morinville, A., Hoffert, C., Hubatsch, D., O'Donnell, D., et al. (2001a). Immunohistochemical distribution of delta opioid receptors in the rat central nervous system: evidence for somatodendritic labeling and antigen-specific cellular compartmentalization. J. Comp. Neurol. 440, 65-84. doi: 10.1002/cne.1370

Cahill, C. M., Morinville, A., Lee, M. C., Vincent, J. P., Collier, B., and Beaudet, A. (2001b). Prolonged morphine treatment targets delta opioid receptors to neuronal plasma membranes and enhances delta-mediated antinociception. J. Neurosci. 21, 7598-7607. doi: 10.1523/JNEUROSCI.21-19-07598. 2001

Cai, X., Huang, H., Kuzirian, M. S., Snyder, L. M., Matsushita, M., Lee, M. C., et al. (2016). Generation of a KOR-Cre knockin mouse strain to study cells involved in kappa opioid signaling. Genesis 54, 29-37. doi: 10.1002/dvg.22910

Ceredig, R. A., and Massotte, D. (2015). Fluorescent knock-in mice to decipher the physiopathological role of $\mathrm{G}$ protein-coupled receptors. Front. Pharmacol. 5:289. doi: $10.3389 /$ fphar.2014.00289
Chabot-Doré, A.-J., Schuster, D. J., Stone, L. S., and Wilcox, G. L. (2015). Analgesic synergy between opioid and $\alpha 2$-adrenoceptors. Br. J. Pharmacol. 172, 388-402. doi: 10.1111/bph.12695

Chan, F., Bradley, A., Wensel, T. G., and Wilson, J. H. (2004). Knock-in human rhodopsin-GFP fusions as mouse models for human disease and targets for gene therapy. Proc. Natl. Acad. Sci. U.S.A. 101, 9109-9114. doi: 10.1073/pnas. 0403149101

Chen, C., Willhouse, A. H., Huang, P., Ko, N., Wang, Y., Xu, B., et al. (2020). Characterization of a knock-in mouse line expressing a fusion protein of $\kappa$ opioid receptor conjugated with tdtomato: 3 -dimensional brain imaging via clarity. eNeuro 7, 1-18. doi: 10.1523/ENEURO.0028-20.2020

Chou, W. Y., Yang, L. C., Lu, H. F., Ko, J. Y., Wang, C. H., Lin, S. H., et al. (2006). Association of $\mu$-opioid receptor gene polymorphism (A118G) with variations in morphine consumption for analgesia after total knee arthroplasty. Acta Anaesthesiol. Scand. 50, 787-792. doi: 10.1111/j.1399-6576.2006.01058.x

Clark, S. D., and Abi-Dargham, A. (2019). The role of dynorphin and the kappa opioid receptor in the symptomatology of schizophrenia: a review of the evidence. Biol. Psychiatry 86, 502-511. doi: 10.1016/j.biopsych.2019.05.012

Codd, E. E., Carson, J. R., Colburn, R. W., Stone, D. J., van Besien, C. R., Zhang, S.-P., et al. (2009). JNJ-20788560 [9-(8-azabicyclo[3.2.1]oct-3-ylidene)9H-xanthene-3-carboxylic acid diethylamide], a selective delta opioid receptor agonist, is a potent and efficacious antihyperalgesic agent that does not produce respiratory depression, pharmacologic toler. J. Pharmacol. Exp. Therap. 329, 241-251. doi: 10.1124/jpet.108.146969

Degrandmaison, J., Abdallah, K., Blais, V., Génier, S., Lalumière, M.-P., Bergeron, F., et al. (2020). In vivo mapping of a GPCR interactome using knockin mice. Proc. Natl. Acad. Sci. U.S.A. 117, 201917906. doi: 10.1073/pnas.1917906117

Degrandmaison, J., Grisé, O., Parent, J. L., and Gendron, L. (2021). Differential barcoding of opioid receptors trafficking. J. Neurosci. Res. 100, 99-128. doi: 10.1002/jnr.24949

Derouiche, L., Pierre, F., Doridot, S., Ory, S., and Massotte, D. (2020). Heteromerization of endogenous $\mathrm{mu}$ and delta opioid receptors induces ligand-selective co-targeting to lysosomes. Molecules 25:4493. doi: 10.3390/ molecules 25194493

Dierich, A., and Kieffer, B. L. (2004). Knockout mouse models in pain research. Methods Mol. Med. 99, 269-299. doi: 10.1385/1-59259-770-X:269

Donica, C. L., Awwad, H. O., Thakker, D. R., and Standifer, K. M. (2013). Cellular mechanisms of nociceptin/orphanin FQ (N/OFQ) peptide (NOP) receptor regulation and heterologous regulation by N/OFQ. Mol. Pharmacol. 83, 907918. doi: $10.1124 / \mathrm{mol} .112 .084632$

Doyle, A., McGarry, M. P., Lee, N. A., and Lee, J. J. (2012). The construction of transgenic and gene knockout/knockin mouse models of human disease. Transgenic Res. 21, 327-349. doi: 10.1007/s11248-011-9537-3

Drakenberg, K., Nikoshkov, A., Horváth, M. C., Fagergren, P., Gharibyan, A., Saarelainen, K., et al. (2006). Mu opioid receptor A118G polymorphism in association with striatal opioid neuropeptide gene expression in heroin abusers. Proc. Natl. Acad. Sci. U.S.A. 103, 7883-7888. doi: 10.1073/pnas.0600871103

Dripps, I. J., Bertels, Z., Moye, L. S., Tipton, A. F., Siegersma, K., Baca, S. M., et al. (2020). Forebrain delta opioid receptors regulate the response of delta agonist in models of migraine and opioid-induced hyperalgesia. Sci. Rep. 10:17629. doi: 10.1038/s41598-020-74605-9

Dykstra, L. A., Gmerek, D. E., Winger, G., and Woods, J. H. (1987). Kappa opioids in rhesus monkeys. I. Diuresis, sedation, analgesia and discriminative stimulus effects. J. Pharmacol. Exp. Therap. 242, 413-420.

Ehrlich, A. T., Semache, M., Gross, F., da Fonte, D. F., Runtz, L., Colley, C., et al. (2019). Biased signaling of the mu opioid receptor revealed in native neurons. iScience 14, 47-57. doi: 10.1016/j.isci.2019.03.011

Erbs, E., Faget, L., Scherrer, G., Kessler, P., Hentsch, D., Vonesch, J. L., et al. (2012). Distribution of delta opioid receptor-expressing neurons in the mouse hippocampus. Neuroscience 221, 203-213. doi: 10.1016/j.neuroscience.2012.06. 023

Erbs, E., Faget, L., Scherrer, G., Matifas, A., Filliol, D., Vonesch, J. L., et al. (2015). A mu-delta opioid receptor brain atlas reveals neuronal co-occurrence in subcortical networks. Brain Struct. Funct. 220, 677-702. doi: 10.1007/s00429014-0717-9

Esteoulle, L., Daubeuf, F., Collot, M., Riché, S., Durroux, T., Brasse, D., et al. (2020). A near-infrared fluorogenic dimer enables background-free imaging 
of endogenous GPCRs in living mice. Chem. Sci. 11, 6824-6829. doi: 10.1039/ d0sc01018a

Evans, C. J., Keith, D. E., Morrison, H., Magendzo, K., and Edwards, R. H. (1992). Cloning of a delta opioid receptor by functional expression. Science 258, 1952-1955. doi: 10.1126/science. 1335167

Feng, P., Rahim, R. T., Cowan, A., Liu-Chen, L. Y., Peng, X., Gaughan, J., et al. (2006). Effects of mu, kappa or delta opioids administered by pellet or pump on oral Salmonella infection and gastrointestinal transit. Eur. J. Pharmacol. 534, 250-257. doi: 10.1016/j.ejphar.2006.01.048

Ferrando, R. E., Newton, K., Chu, F., Webster, J. D., and French, D. M. (2015). Immunohistochemical detection of FLAG-tagged endogenous proteins in knock-in mice. J. Histochem. Cytochem. 63, 244-255. doi: 10.1369/ 0022155414568101

Fraser, G. L., Gaudreau, G. A., Clarke, P. B., Ménard, D. P., and Perkins, M. N. (2000). Antihyperalgesic effects of delta opioid agonists in a rat model of chronic inflammation. Br. J. Pharmacol. 129, 1668-1672. doi: 10.1038/sj.bjp.0703248

Friedman, A., Homma, D., Gibb, L. G., Amemori, K. I., Rubin, S. J., Hood, A. S., et al. (2015). A corticostriatal path targeting striosomes controls decisionmaking under conflict. Cell 161, 1320-1333. doi: 10.1016/j.cell.2015.04.049

Fritzwanker, S., Moulédous, L., Mollereau, C., Froment, C., Burlet-Schiltz, O., Effah, F., et al. (2021). HA-MOP knockin mice express the canonical $\mu$-opioid receptor but lack detectable splice variants. Commun. Biol. 4:1070. doi: 10.1038/ s42003-021-02580-6

Fukuda, K., Kato, S., Mori, K., Nishi, M., Takeshima, H., Iwabe, N., et al. (1994). cDNA cloning and regional distribution of a novel member of the opioid receptor family. FEBS Lett. 343, 42-46. doi: 10.1016/0014-5793(94)80603-9

Gaj, T., Gersbach, C. A., and Barbas, C. F. (2013). ZFN, TALEN, and CRISPR/Casbased methods for genome engineering. Trends Biotechnol. 31, 397-405. doi: 10.1016/j.tibtech.2013.04.004

Gallantine, E. L., and Meert, T. F. (2005). A comparison of the antinociceptive and adverse effects of the mu-opioid agonist morphine and the delta-opioid agonist SNC80. Basic Clin. Pharmacol. Toxicol. 97, 39-51. doi: 10.1111/j.17427843.2005.pto_97107.x

Gardon, O., Faget, L., Chu Sin Chung, P., Matifas, A., Massotte, D., and Kieffer, B. L. (2014). Expression of mu opioid receptor in dorsal diencephalic conduction system: new insights for the medial habenula. Neuroscience 277, 595-609. doi: 10.1016/j.neuroscience.2014.07.053

Gelernter, J., and Kranzler, H. R. (2000). Variant detection at the $\delta$ opioid receptor (OPRD1) locus and population genetics of a novel variant affecting protein sequence. Hum. Genet. 107, 86-88. doi: 10.1007/s004390000340

Gendron, L., Cahill, C. M., von Zastrow, M., Schiller, P. W., and Pineyro, G. (2016). Molecular pharmacology of $\delta$-Opioid receptors. Pharmacol. Rev. 68, 631-700. doi: 10.1124/pr.114.008979

Gendron, L., Lucido, A. L., Mennicken, F., O’Donnell, D., Vincent, J.-P., Stroh, T., et al. (2006). Morphine and pain-related stimuli enhance cell surface availability of somatic delta-opioid receptors in rat dorsal root ganglia. J. Neurosci. 26, 953-962. doi: 10.1523/JNEUROSCI.3598-05.2006

Gillis, A., Kliewer, A., Kelly, E., Henderson, G., Christie, M. J., Schulz, S., et al. (2020). Critical Assessment of G protein-biased agonism at the $\mu$-Opioid receptor. Trends Pharmacol. Sci. 41, 947-959. doi: 10.1016/j.tips.2020.09.009

Goth, C. K., Petäjä-Repo, U. E., and Rosenkilde, M. M. (2020). G Proteincoupled receptors in the sweet spot: glycosylation and other post-translational modifications. ACS Pharmacol. Transl. Sci. 3, 237-245. doi: 10.1021/acsptsci. 0c00016

Grecksch, G., Just, S., Pierstorff, C., Imhof, A. K., Glück, L., Doll, C., et al. (2011). Analgesic tolerance to high-efficacy agonists but not to morphine is diminished in phosphorylation-deficient S375A $\mu$-opioid receptor knock-in mice. J. Neurosci. 31, 13890-13896.

Grisel, J. E., Mogil, J. S., Belknap, J. K., and Grandy, D. K. (1996). Orphanin FQ acts as a supraspinal, but not a spinal, anti-opioid peptide. NeuroReport 7 , 2125-2129. doi: 10.1097/00001756-199609020-00012

Guerrero-Alba, R., Valdez-Morales, E. E., Jiménez-Vargas, N. N., Bron, R., Poole, D., Reed, D., et al. (2018). Co-expression of $\mu$ and $\delta$ opioid receptors by mouse colonic nociceptors. Br. J. Pharmacol. 175, 2622-2634. doi: 10.1111/bph.14222

Hall, B., Cho, A., Limaye, A., Cho, K., Khillan, J., and Kulkarni, A. B. (2018). Genome editing in mice using CRISPR/Cas9 technology. Curr. Protoc. Cell Biol. 81:e57. doi: $10.1002 / \mathrm{cpcb} .57$
Hauser, A. S., Attwood, M. M., Rask-Andersen, M., Schiöth, H. B., and Gloriam, D. E. (2017). Trends in GPCR drug discovery: new agents, targets and indications. Nat. Rev. Drug Discov. 16, 829-842. doi: 10.1038/nrd.2017.178

He, X., Sandhu, H. K., Yang, Y., Hua, F., Belser, N., Kim, D. H., et al. (2013). Neuroprotection against hypoxia/ischemia: $\delta$-opioid receptor-mediated cellular/molecular events. Cell. Mol. Life Sci. 70, 2291-2303. doi: 10.1007/ s00018-012-1167-2

Headrick, J. P., See Hoe, L. E., du Toit, E. F., and Peart, J. N. (2015). Opioid receptors and cardioprotection - "opioidergic conditioning" of the heart. $\mathrm{Br}$. J. Pharmacol. 172, 2026-2050. doi: 10.1111/bph.13042

Hilderbrand, S. A., and Weissleder, R. (2010). Near-infrared fluorescence: application to in vivo molecular imaging. Curr. Opin. Chem. Biol. 14, 71-79. doi: 10.1016/j.cbpa.2009.09.029

Holdridge, S. V., and Cahill, C. M. (2007). Spinal administration of a $\delta$ opioid receptor agonist attenuates hyperalgesia and allodynia in a rat model of neuropathic pain. Eur. J. Pain 11, 685-693. doi: 10.1016/j.ejpain.2006.10.008

Hsu, P. D., Lander, E. S., and Zhang, F. (2014). Development and applications of CRISPR-Cas9 for genome engineering. Cell 157, 1262-1278. doi: 10.1016/j.cell. 2014.05.010

Huang, P., Chen, C., Mague, S. D., Blendy, J. A., and Liu-Chen, L. Y. (2012). A common single nucleotide polymorphism A118G of the $\mu$ opioid receptor alters its N-glycosylation and protein stability. Biochem. J. 441, 379-386. doi: 10.1042/BJ20111050

Huang, P., Chen, C., Xu, W., Yoon, S. I., Unterwald, E. M., Pintar, J. E., et al. (2008). Brain region-specific $\mathrm{N}$-glycosylation and lipid rafts association of the rat mu opioid receptor. Biochem. Biophys. Res. Commun. 365, 82-88. doi: 10.1016/j. bbrc.2007.10.128

Ingvar, M. (1999). Pain and functional imaging. Philos. Trans. R. Soc. Lond. Ser. B Biol. Sci. 354, 1347-1358. doi: 10.1098/rstb.1999.0483

Jacobson, M. L., Browne, C. A., and Lucki, I. (2019). Kappa opioid receptor antagonists as potential therapeutics for stress-related disorders. Annu. Rev. Pharmacol. Toxicol. 60, 615-636. doi: 10.1146/annurev-pharmtox-010919023317

Jo, M., and Jung, S. T. (2016). Engineering therapeutic antibodies targeting G-protein-coupled receptors. Exp. Mol. Med. 48:e207. doi: 10.1038/emm.2015. 105

Joubert, F., Guerrero-Moreno, A., Fakih, D., Reboussin, E., Gaveriaux-Ruff, C., Acosta, M. C., et al. (2020). Topical treatment with a mu opioid receptor agonist alleviates corneal allodynia and corneal nerve sensitization in mice. Biomed. Pharmacother. 132:110794. doi: 10.1016/j.biopha.2020.110794

Joung, J. K., and Sander, J. D. (2013). TALENs: a widely applicable technology for targeted genome editing. Nat. Rev. Mol. Cell Biol. 14, 49-55. doi: 10.1038/ nrm3486

Kamei, J., Kawai, K., Mizusuna, A., Saitoh, A., Morita, K., Narita, M., et al. (1997). Supraspinal $\delta 1$-opioid receptor-mediated antinociceptive properties of (-)-TAN-67 in diabetic mice. Eur. J. Pharmacol. 322, 27-30. doi: 10.1016/s00142999(97)00085-x

Kaneko, T., Minami, M., Satoh, M., and Mizuno, N. (1995). Immunocytochemical localization of $\mu$-opioid receptor in the rat caudate-putamen. Neurosci. Lett. 184, 149-152. doi: 10.1016/0304-3940(94)11192-1

Kieffer, B. L. (1999). Opioids: first lessons from knockout mice. Trends Pharmacol. Sci. 20, 19-26. doi: 10.1016/s0165-6147(98)01279-6

Kieffer, B. L., Befort, K., Gaveriaux-Ruff, C., and Hirth, C. G. (1992). The delta-opioid receptor: isolation of a cDNA by expression cloning and pharmacological characterization. Proc. Natl. Acad. Sci. U.S.A. 89, 1204812052.

Kim, S. Y., Cho, J. H., Murray, E., Bakh, N., Choi, H., Ohn, K., et al. (2015). Stochastic electrotransport selectively enhances the transport of highly electromobile molecules. Proc. Natl. Acad. Sci. U.S.A. 112, E6274-E6283. doi: 10.1073/pnas.1510133112

Kleeman, B., Olsson, A., Newkold, T., Kofron, M., DeLay, M., Hildeman, D., et al. (2018). A guide to choosing fluorescent protein combinations for flow cytometric analysis based on spectral overlap. Cytometry Part A 93, 556-562. doi: $10.1002 /$ cyto.a. 23360

Knežević, A., Polašek, O., Gornik, O., Rudan, I., Campbell, H., Hayward, C., et al. (2009). Variability, heritability and environmental determinants of human plasma N-glycome. J. Proteome Res. 8, 694-701. doi: 10.1021/pr800737u 
Ko, J. L., Arvidsson, U., Williams, F. G., Law, P. Y., Elde, R., and Loh, H. H. (1999). Visualization of time-dependent redistribution of $\delta$-opioid receptors in neuronal cells during prolonged agonist exposure. Mol. Brain Res. 69, 171-185. doi: 10.1016/s0169-328x(99)00094-7

Kono, M., Conlon, E. G., Lux, S. Y., Yanagida, K., Hla, T., and Proia, R. L. (2017). Bioluminescence imaging of $\mathrm{G}$ protein-coupled receptor activation in living mice. Nat. Commun. 8:1163. doi: 10.1038/s41467-017-01340-7

Kooistra, A. J., Mordalski, S., Pándy-Szekeres, G., Esguerra, M., Mamyrbekov, A., Munk, C., et al. (2021). GPCRdb in 2021: integrating GPCR sequence, structure and function. Nucleic Acids Res. 49, D335-D343. doi: 10.1093/nar/gkaa1080

Kreek, M. J., Bart, G., Lilly, C., Laforge, K. S., and Nielsen, D. A. (2005). Pharmacogenetics and human molecular genetics of opiate and cocaine addictions and their treatments. Pharmacol. Rev. 57, 1-26. doi: 10.1124/pr.57.1. 1

Land, B. B., Bruchas, M. R., Schattauer, S., Giardino, W. J., Aita, M., Messinger, D., et al. (2009). Activation of the kappa opioid receptor in the dorsal raphe nucleus mediates the aversive effects of stress and reinstates drug seeking. Proc. Natl. Acad. Sci. U.S.A. 106, 19168-19173. doi: 10.1073/pnas.0910705106

Lemos Duarte, M., and Devi, L. A. (2020). Post-translational modifications of opioid receptors. Trends Neurosci. 43, 417-432. doi: 10.1016/j.tins.2020.03.011

Lerman, C., Wileyto, E. P., Patterson, F., Rukstalis, M., Audrain-McGovern, J., Restine, S., et al. (2004). The functional mu opioid receptor (OPRM1) Asn40Asp variant predicts short-term response to nicotine replacement therapy in a clinical trial. Pharmacogenomics J. 4, 184-192. doi: 10.1038/sj.tpj.6500238

Li, Z., Proud, D., Zhang, C., Wiehler, S., and McDougall, J. J. (2005). Chronic arthritis down-regulates peripheral $\mu$-opioid receptor expression with concomitant loss of endomorphin 1 antinociception. Arthritis Rheum. 52, 3210-3219. doi: 10.1002/art.21359

Lu, R., Li, Y., Zhang, Y., Chen, Y., Shields, A. D., Winder, D. G., et al. (2009). Epitope-tagged receptor knock-in mice reveal that differential desensitization of $\alpha 2$-adrenergic responses is because of ligand-selective internalization. J. Biol. Chem. 284, 13233-13243. doi: 10.1074/jbc.M807535200

Lucido, A. L., Morinville, A., Gendron, L., Stroh, T., and Beaudet, A. (2005). Prolonged morphine treatment selectively increases membrane recruitment of $\delta$-opioid receptors in mouse basal ganglia. J. Mol. Neurosci. 25, 207-213. doi: $10.1385 / \mathrm{JMN}: 25: 3: 207$

Maduna, T., Audouard, E., Dembélé, D., Mouzaoui, N., Reiss, D., Massotte, D., et al. (2019). Microglia express mu opioid receptor: insights from transcriptomics and fluorescent reporter mice. Front. Psychiatry 10:726. doi: 10.3389/fpsyt.2018. 00726

Madziva, M. T., and Michael, J. (2001). Trafficking of green fluorescent proteintagged muscarinic M4 receptors in NG108-15 cells Eur. J. Pharmacol. 428, 9-18. doi: 10.1016/s0014-2999(01)01266-3

Mague, S. D., Isiegas, C., Huang, P., Liu-Chen, L.-Y., Lerman, C., and Blendy, J. A. (2009). Mouse model of OPRM1 (A118G) polymorphism has sex-specific effects on drug-mediated behavior. Proc. Natl. Acad. Sci. U.S.A. 106, 1084710852. doi: 10.1073/pnas.0901800106

Maldonado, R., Cabañero, D., and Baños, J. E. (2018). Usefulness of knockout mice to clarify the role of the opioid system in chronic pain. Br. J. Pharmacol. 175:2791. doi: 10.1111/bph.v175.14/issuetoc

Mambretti, E. M., Kistner, K., Mayer, S., Massotte, D., Kieffer, B. L., Hoffmann, C., et al. (2016). Functional and structural characterization of axonal opioid receptors as targets for analgesia. Mol. Pain 12:174480691662873. doi: 10.1177/ 1744806916628734

Mann, A., Moulédous, L., Froment, C., O’neill, P. R., Dasgupta, P., Günther, T., et al. (2019). Agonist-selective NOP receptor phosphorylation correlates in vitro and in vivo and reveals differential post-activation signaling by chemically diverse agonists Sci. Signal. 12:eaau8072. doi: 10.1126/scisignal.aau8072

Mansour, A., Fox, C. A., Burke, S., Akil, H., and Watson, S. J. (1995). Immunohistochemical localization of the cloned $\mu$ opioid receptor in the rat CNS. J. Chem. Neuroanat. 8, 283-305. doi: 10.1016/0891-0618(95)00055-c

Märtin, A., Calvigioni, D., Tzortzi, O., Fuzik, J., Wärnberg, E., and Meletis, K. (2019). A spatiomolecular map of the striatum. Cell Rep. 29, 4320-4333e5. doi: 10.1016/j.celrep.2019.11.096

Matsuhashi, H., Horii, Y., and Kato, K. (2003). Region-specific and epileptogenicdependent expression of six subtypes of alpha2,3-sialyltransferase in the adult mouse brain. J. Neurochem. 84, 53-66. doi: 10.1046/j.1471-4159.2003.01 257.x
McDonald, N. A., Henstridge, C. M., Connolly, C. N., and Irving, A. J. (2007). Generation and functional characterization of fluorescent, N-terminally tagged CB1 receptor chimeras for live-cell imaging. Mol. Cell. Neurosci. 35, 237-248. doi: 10.1016/j.mcn.2007.02.016

McDougall, J. J., Barin, A. K., and McDougall, C. M. (2004). Loss of vasomotor responsiveness to the $\mu$-opioid receptor ligand endomorphin-1 in adjuvant monoarthritic rat knee joints. Am. J. Physiol. Regul. Integr. Comp. Physiol. 286, R634-R641. doi: 10.1152/ajpregu.00464.2003

McLean, A. J., and Milligan, G. (2000). Ligand regulation of green fluorescent protein-tagged forms of the human beta(1)- and beta(2)-adrenoceptors; comparisons with the unmodified receptors. Br. J. Pharmacol. 130, 1825-1832. doi: 10.1038/sj.bjp.0703506

Michel, M. C., Wieland, T., and Tsujimoto, G. (2009). How reliable are G-proteincoupled receptor antibodies? Naunyn Schmiedebergs Arch. Pharmacol. 379, 385-388.

Mogil, J. S., and Pasternak, G. W. (2001). The molecular and behavioral pharmacology of the orphanin FQ/nociceptin peptide and receptor family. Pharmacol. Rev. 53, 381-415.

Mogil, J. S., Grisel, J. E., Reinscheid, R. K., Civelli, O., Belknap, J. K., and Grandy, D. K. (1996a). Orphanin FQ is a functional anti-opioid peptide. Neuroscience 75, 333-337. doi: 10.1016/0306-4522(96)00338-7

Mogil, J. S., Grisel, J. E., Zhangs, G., Belknap, J. K., and Grandy, D. K. (1996b). Functional antagonism of $\mu-, \delta$ - and $\kappa$-opioid antinociception by orphanin FQ. Neurosci. Lett. 214, 131-134.

Mollereau, C., Parmentier, M., Mailleux, P., Butour, J. L., Moisand, C., Chalon, P., et al. (1994). ORL1, a novel member of the opioid receptor family. Cloning, functional expression and localization. FEBS Lett. 341, 33-38. doi: 10.1016/ 0014-5793(94)80235- 1

Morgan, M. M., and Christie, M. J. (2011). Analysis of opioid efficacy, tolerance, addiction and dependence from cell culture to human. Br. J. Pharmacol. 164, 1322-1334. doi: 10.1111/j.1476-5381.2011.01335.x

Mousa, S. A., Shaqura, M., Khalefa, B. I., Zöllner, C., Schaad, L., Schneider, J., et al. (2013). Rab7 silencing prevents $\mu$-opioid receptor lysosomal targeting and rescues opioid responsiveness to strengthen diabetic neuropathic pain therapy. Diabetes 62, 1308-1319. doi: 10.2337/db12-0590

Murali, S. S., Napier, I. A., Rycroft, B. K., and Christie, M. J. (2012). Opioidrelated (ORL1) receptors are enriched in a subpopulation of sensory neurons and prolonged activation produces no functional loss of surface $\mathrm{N}$-type calcium channels. J. Physiol. 590, 1655-1667. doi: 10.1113/jphysiol.2012.228429

Nadal, X., La Porta, C., Bura, S. A., and Maldonado, R. (2013). Involvement of the opioid and cannabinoid systems in pain control: new insights from knockout studies. Eur. J. Pharmacol. 716, 142-157. doi: 10.1016/j.ejphar.2013.01.077

Nagai, T., Ibata, K., Park, E. S., Kubota, M., Mikoshiba, K., and Miyawaki, A. (2002). A variant of yellow fluorescent protein with fast and efficient maturation for cell-biological applications. Nat. Biotechnol. 20, 87-90. doi: 10.1038/nbt0102-87

Nam, M. H., Han, K. S., Lee, J., Bae, J. Y., An, H., Park, S., et al. (2018). Expression of $\mu$-opioid receptor in CA1 hippocampal astrocytes. Exp. Neurobiol. 27, 120-128. doi: 10.5607/en.2018.27.2.120

Neal, C. R., Mansour, A., Reinscheid, R., Nothacker, H.-P., Civelli, O., Akil, H., et al. (1999). Opioid receptor-like (ORL1) receptor distribution in the rat central nervous system: comparison of ORL1 receptor mRNA expression with (125)I[(14)Tyr]-orphanin FQ binding. J. Comp. Neurol. 412, 563-605. doi: 10.1002/ (sici) 1096-9861(19991004)412:4<563::aid-cne2>3.0.co;2-z

Nozaki, C., Nagase, H., Nemoto, T., Matifas, A., Kieffer, B. L., and Gaveriaux-Ruff, C. (2014). In vivo properties of KNT-127, a novel $\delta$ opioid receptor agonist: receptor internalization, antihyperalgesia and antidepressant effects in mice. $\mathrm{Br}$. J. Pharmacol. 171, 5376-5386. doi: 10.1111/bph.12852

Okunomiya, T., Hioki, H., Nishimura, C., Yawata, S., Imayoshi, I., Kageyama, R., et al. (2020). Generation of a MOR-CreER knock-in mouse line to study cells and neural circuits involved in mu opioid receptor signaling. Genesis 58, e23341. doi: $10.1002 / \mathrm{dvg} .23341$

Oliinyk, O. S., Shemetov, A. A., Pletnev, S., Shcherbakova, D. M., and Verkhusha, V. V. (2019). Smallest near-infrared fluorescent protein evolved from cyanobacteriochrome as versatile tag for spectral multiplexing. Nat. Commun. 10:279. doi: 10.1038/s41467-018-08050-8

Otis, V., Sarret, P., and Gendron, L. (2011). Spinal activation of delta opioid receptors alleviates cancer-related bone pain. Neuroscience 183, 221-229. doi: 10.1016/j.neuroscience.2011.03.052 
Ozawa, A., Brunori, G., Cippitelli, A., Toll, N., Schoch, J., Kieffer, B. L., et al. (2018). Analysis of the distribution of spinal NOP receptors in a chronic pain model using NOP-eGFP knock-in mice. Br. J. Pharmacol. 175, 2662-2675. doi: $10.1111 /$ bph.14225

Ozawa, A., Brunori, G., Mercatelli, D., Wu, J., Cippitelli, A., Zou, B., et al. (2015). Knock-in mice with NOP-eGFP receptors identify receptor cellular and regional localization. J. Neurosci. 35, 11682-11693. doi: 10.1523/JNEUROSCI. 5122-14.2015

Pasquini, F., Bochet, P., Garbay-Jaureguiberry, C., Roques, B. P., Rossier, J., and Beaudet, A. (1992). Electron microscopic localization of photoaffinity-labelled delta opioid receptors in the neostriatum of the rat. J. Comp. Neurol. 326, 229-244. doi: 10.1002/cne.903260206

Pasternak, G. W., and Pan, Y. X. (2013). Mu opioids and their receptors: evolution of a concept. Pharmacol. Rev. 65, 1257-1317. doi: 10.1124/pr.112.007138

Pathan, H., and Williams, J. (2012). Basic opioid pharmacology: an update. Br. J. Pain 6, 11-16. doi: 10.1177/2049463712438493

Paton, K. F., Atigari, D. V., Kaska, S., Prisinzano, T., and Kivell, B. M. (2020). Strategies for developing $\mathrm{k}$ opioid receptor agonists for the treatment of pain with fewer side effects. J. Pharmacol. Exp. Therap. Am. Soc. Pharmacol. Exp. Ther. 375, 332-348. doi: 10.1124/jpet.120.000134

Pert, C. B., Kuhar, M. J., and Snyder, S. H. (1976). Opiate receptor: autoradiographic localization in rat brain. Proc. Natl. Acad. Sci. U.S.A. 73, 3729-3733. doi: 10.1073/pnas.73.10.3729

Pfeiffer, A., Brantl, V., Herz, A., and Emrich, H. M. (1986). Psychotomimesis mediated by kappa opiate receptors. Science (New York, NY) 233, 774-776. doi: 10.1126/science.3016896

Pradhan, A. A. A., Becker, J. A. J., Scherrer, G., Tryoen-Toth, P., Filliol, D., Matifas, A., et al. (2009). In vivo delta opioid receptor internalization controls behavioral effects of agonists. PLoS One 4:e5425. doi: 10.1371/journal.pone.0005425

Pradhan, A. A. A., Walwyn, W., Nozaki, C., Filliol, D., Erbs, E., Matifas, A., et al. (2010). Ligand-directed trafficking of the $\delta$-opioid receptor in vivo: two paths toward analgesic tolerance. J. Neurosci. 30, 16459-16468. doi: 10.1523/ JNEUROSCI.3748-10.2010

Ramchandani, V. A., Umhau, J., Pavon, F. J., Ruiz-Velasco, V., Margas, W., Sun, H., et al. (2011). A genetic determinant of the striatal dopamine response to alcohol in men. Mol. Psychiatry 16, 809-817. doi: 10.1038/mp.2010.56

Rau, K. K., Caudle, R. M., Cooper, B. Y., and Johnson, R. D. (2005). Diverse immunocytochemical expression of opioid receptors in electrophysiologically defined cells of rat dorsal root ganglia. J. Chem. Neuroanat. 29, 255-264. doi: 10.1016/j.jchemneu.2005.02.002

Ray, L. A., and Hutchison, K. E. (2004). A polymorphism of the mu-opioid receptor gene (OPRM1) and sensitivity to the effects of alcohol in humans. Alcohol. Clin. Exp. Res. 28, 1789-1795. doi: 10.1097/01.alc.0000148114.34000.b9

Ray, L. A., and Hutchison, K. E. (2007). Effects of naltrexone on alcohol sensitivity and genetic moderators of medication response: a double-blind placebocontrolled study. Arch. Gen. Psychiatry 64, 1069-1077. doi: 10.1001/archpsyc. 64.9.1069

Reed, B., Butelman, E. R., Fry, R. S., Kimani, R., and Kreek, M. J. (2018). Repeated administration of opra kappa (LY2456302), a novel, short-acting, selective KOP-r antagonist, in persons with and without cocaine dependence. Neuropsychopharmacology 43, 739-750. doi: 10.1038/npp.2017.205

Reinscheid, R. K., Nothacker, H. P., Bourson, A., Ardati, A., Henningsen, R. A., Bunzow, J. R., et al. (1995). Orphanin FQ: a neuropeptide that activates an opioidlike G protein-coupled receptor. Science 270, 792-794. doi: 10.1126/ science.270.5237.792

Rezaï, X., Faget, L., Bednarek, E., Schwab, Y., Kieffer, B. L., and Massotte, D. (2012). Mouse delta opioid receptors are located on presynaptic afferents to hippocampal pyramidal cells. Cell. Mol. Neurobiol. 32, 509-516. doi: 10.1007/ s10571-011-9791-1

Rios, C., Gomes, I., and Devi, L. A. (2004). Interactions between delta opioid receptors and alpha-adrenoceptors. Clin. Exp. Pharmacol. Physiol. 31, 833-836. doi: 10.1111/j.1440-1681.2004.04076.x

Roth, B. L., Baner, K., Westkaemper, R., Siebert, D., Rice, K. C., Steinberg, S., et al. (2002). Salvinorin A: a potent naturally occurring nonnitrogenous kappa opioid selective agonist. Proc. Natl. Acad. Sci. U.S.A. 99, 11934-11939. doi: $10.1073 /$ pnas. 182234399

Saitoh, A., Sugiyama, A., Yamada, M., Inagaki, M., Oka, J. I., Nagase, H., et al. (2013). The novel $\delta$ opioid receptor agonist KNT-127 produces distinct anxiolytic-like effects in rats without producing the adverse effects associated with benzodiazepines. Neuropharmacology 67, 485-493. doi: 10 . 1016/j.neuropharm.2012.11.025

Schäfer, K., and Braun, T. (1995). Monoclonal anti-FLAG antibodies react with a new isoform of rat $\mathrm{Mg} 2+$ dependent protein phosphatase beta. Biochem. Biophys. Res. Commun. 207, 708-714. doi: 10.1006/bbrc.1995.1245

Scherrer, G., Tryoen-Toth, P., Filliol, D., Matifas, A., Laustriat, D., Cao, Y. Q., et al. (2006). Knockin mice expressing fluorescent delta-opioid receptors uncover G protein-coupled receptor dynamics in vivo. Proc. Natl. Acad. Sci. U.S.A. 103, 9691-9696. doi: 10.1073/pnas.0603359103

Shiwarski, D. J., Darr, M., Telmer, C. A., Bruchez, M. P., and Puthenveedu, M. A. (2017a). PI3K class II $\alpha$ regulates $\delta$-opioid receptor export from the trans -Golgi network. Mol. Biol. Cell 28, 2202-2219. doi: 10.1091/mbc.E17-01-0030

Shiwarski, D. J., Tipton, A., Giraldo, M. D., Schmidt, B. F., Gold, M. S., Pradhan, A. A., et al. (2017b). A PTEN-regulated checkpoint controls surface delivery of $\delta$ opioid receptors. J. Neurosci. 37, 3741-3752. doi: 10.1523/JNEUROSCI.292316.2017

Sia, A. T., Lim, Y., Lim, E. C. P., Goh, R. W. C., Law, H. Y., Landau, R., et al. (2008). A118G single nucleotide polymorphism of human mu-opioid receptor gene influences pain perception and patient-controlled intravenous morphine consumption after Intrathecal morphine for Postcesarean analgesia Anesthesiology 109, 520-526. doi: 10.1097/ALN.0b013e318182af21

Siuda, E. R., Copits, B. A., Schmidt, M. J., Baird, M. A., Al-Hasani, R., Planer, W. J., et al. (2015). Spatiotemporal control of opioid signaling and behavior. Neuron 86, 923-935. doi: 10.1016/j.neuron.2015.03.066

Stanta, J. L., Saldova, R., Struwe, W. B., Byrne, J. C., Leweke, F. M., Rothermund, M., et al. (2010). Identification of N-glycosylation changes in the CSF and serum in patients with schizophrenia. J. Proteome Res. 9, 4476-4489. doi: 10.1021/ pr1002356

Stolik, S., Delgado, J. A., Pérez, A., and Anasagasti, L. (2000). Measurement of the penetration depths of red and near infrared light in human "ex vivo" tissues. J. Photochem. Photobiol. B Biol. 57, 90-93. doi: 10.1016/s1011-1344(00)00082-8

Su, D., Wang, M., Ye, C., Fang, J., Duan, Y., Zhang, Z., et al. (2017). Onestep generation of mice carrying a conditional allele together with an HA-tag insertion for the delta opioid receptor. Sci. Rep. 7:44476. doi: 10.1038/srep44476

Tan, K. R., Yvon, C., Turiault, M., Mirzabekov, J. J., Doehner, J., Labouèbe, G., et al. (2012). GABA neurons of the VTA drive conditioned place aversion. Neuron 73, 1173-1183. doi: 10.1016/j.neuron.2012.02.015

Tanowitz, M., and von Zastrow, M. A. (2003). Novel endocytic recycling signal that distinguishes the membrane trafficking of naturally occurring opioid receptors. J. Biol. Chem. 278, 45978-45986. doi: 10.1074/jbc.M304504200

Tanowitz, M., Hislop, J. N., and von Zastrow, M. (2008). Alternative splicing determines the post-endocytic sorting fate of G-protein-coupled receptors. J. Biol. Chem. 283, 35614-35621. doi: 10.1074/jbc.M806588200

Tsao, P. I., and von Zastrow, M. (2000). Type-specific sorting of G protein-coupled receptors after endocytosis. J. Biol. Chem. 275, 11130-11140. doi: 10.1074/jbc. 275.15.11130

van den Wildenberg, E., Wiers, R. W., Dessers, J., Janssen, R. G. J. H., Lambrichs, E. H., Smeets, H. J. M., et al. (2007). A functional polymorphism of the $\mu$ opioid receptor gene (OPRM1) influences cue-induced craving for alcohol in male heavy drinkers. Alcohol. Clin. Exp. Res. 31, 1-10. doi: 10.1111/j.1530-0277. 2006.00258.x

van Unen, J., Woolard, J., Rinken, A., Hoffmann, C., Hill, S. J., Goedhart, J., et al. (2015). A perspective on studying G-protein-coupled receptor signaling with resonance energy transfer biosensors in living organisms. Mol. Pharmacol. 88, 589-595. doi: 10.1124/mol.115.098897

Vardy, E., Robinson, J. E., Li, C., Olsen, R. H. J., DiBerto, J. F., Giguere, P. M., et al. (2015). A new DREADD facilitates the multiplexed chemogenetic interrogation of behavior. Neuron 86, 936-946. doi: 10.1016/j.neuron.2015.03.065

Wang, H. B., Guan, J. S., Bao, L., and Zhang, X. (2008). Distinct subcellular distribution of $\delta$-opioid receptor fused with various tags in PC12 cells. Neurochem. Res. 33, 2028-2034. doi: 10.1007/s11064-008-9678-9

Wang, H. B., Zhao, B., Zhonga, Y. Q., Li, K. C., Li, Z. Y., Wang, Q., et al. (2010). Coexpression of $\delta$ - and $\mu$-opioid receptors in nociceptive sensory neurons. Proc. Natl. Acad. Sci. U.S.A. 107, 13117-13122. doi: 10.1073/pnas.100838 2107

Wang, H., and Pickel, V. M. (2001). Preferential cytoplasmic localization of delta-opioid receptors in rat striatal patches: comparison with plasmalemmal 
mu-opioid receptors. J. Neurosci. 21, 3242-3250. doi: 10.1523/JNEUROSCI.2109-03242.2001

Wang, J. B., Johnson, P. S., Imai, Y., Persico, A. M., Ozenberger, B. A., Eppler, C. M., et al. (1994). cDNA cloning of an orphan opiate receptor gene family member and its splice variant. FEBS Lett. 348, 75-79. doi: 10.1016/0014-5793(94)005575

Wang, W., Loh, H. H., and Law, P. Y. (2003). The intracellular trafficking of opioid receptors directed by carboxyl tail and a di-leucine motif in Neuro2A cells. J. Biol. Chem. 278, 36848-36858. doi: 10.1074/jbc.M301540200

Wang, Y. J., Huang, P., Ung, A., Blendy, J. A., and Liu-Chen, L. Y. (2012). Reduced expression of the mu opioid receptor in some, but not all, brain regions in mice with OPRM1 A112G. Neuroscience 205, 178-184. doi: 10.1016/j.neuroscience. 2011.12.033

Wei, X., Centeno, M. V., Ren, W., Borruto, A. M., Procissi, D., Xu, T., et al. (2021). Activation of the dorsal, but not the ventral, hippocampus relieves neuropathic pain in rodents. Pain 162, 2865-2880. doi: 10.1097/j.pain.0000000000002279

Whistler, J. L., Enquist, J., Marley, A., Fong, J., Gladher, F., Tsuruda, P., et al. (2002). Modulation of postendocytic sorting of G protein-coupled receptors. Science 297, 615-620. doi: 10.1126/science.1073308

Xu, X. J., Hao, J. X., and Wiesenfeld-Hallin, Z. (1996). Nociceptin or antinociceptin: potent spinal antinociceptive effect of orphanin FQ/nociceptin in the rat. Neuroreport 7, 2092-2094.

Yaksh, T. L., and Rudy, T. A. (1976). Analgesia mediated by a direct spinal action of narcotics. Science 192, 1357-1358. doi: 10.1126/science.1273597

Yamamoto, T., Nozaki-Taguchi, N., and Kimura, S. (1997). Analgesic effect of intrathecally administered nociceptin, an opioid receptor-like receptor agonist, in the rat formalin test. Neuroscience 81, 249-254. doi: 10.1016/s0306-4522(97) 00166-8
Yoshizawa, T., Ito, M., and Doya, K. (2018). Reward-predictive neural activities in striatal striosome compartments. eNeuro 5:ENEURO.0367-17.2018. doi: 10. 1523/ENEURO.0367-17.2018

Yuan, L., Lin, W., Zheng, K., He, L., and Huang, W. (2013). Far-red to near infrared analyte-responsive fluorescent probes based on organic fluorophore platforms for fluorescence imaging. Chem. Soc. Rev. 42, 622-661. doi: 10.1039/c2cs35313j

Zhang, X. Y., Dou, Y. N., Yuan, L., Li, Q., Zhu, Y. J., Wang, M., et al. (2020). Different neuronal populations mediate inflammatory pain analgesia by exogenous and endogenous opioids. eLife 9:e55289. doi: 10.7554/eLife.55 289

Conflict of Interest: The authors declare that the research was conducted in the absence of any commercial or financial relationships that could be construed as a potential conflict of interest.

Publisher's Note: All claims expressed in this article are solely those of the authors and do not necessarily represent those of their affiliated organizations, or those of the publisher, the editors and the reviewers. Any product that may be evaluated in this article, or claim that may be made by its manufacturer, is not guaranteed or endorsed by the publisher.

Copyright (C) 2022 Degrandmaison, Rochon-Haché, Parent and Gendron. This is an open-access article distributed under the terms of the Creative Commons Attribution License (CC BY). The use, distribution or reproduction in other forums is permitted, provided the original author(s) and the copyright owner(s) are credited and that the original publication in this journal is cited, in accordance with accepted academic practice. No use, distribution or reproduction is permitted which does not comply with these terms. 